\section{GENRE}

en séries

\section{Genre en séries}

Cinéma, télévision, médias

$11 \mid 2020$

Freaks en tous genres : corps mutants, cyborgs, métamorphoses \& fantastiques

\title{
L'espion au complet gris : masculinité et performance dans La Mort aux trousses
}

Traduction de "The Spy in the Gray Flannel Suit », in Steven cohan, Masked Men : Masculinity and the Movies in the Fifties, Bloomington and Indianapolis, Indiana University Press, 1997, p. 1-33.

\section{Steven Cohan}

Traducteur : Jules Sandeau

\section{OpenEdition}

\section{Journals}

Édition électronique

URL : http://journals.openedition.org/ges/1067

DOI : 10.4000/ges. 1067

ISSN : 2437-6563

Éditeur

Presses universitaires de Bordeaux

\section{Référence électronique}

Steven Cohan, «L'espion au complet gris : masculinité et performance dans La Mort aux trousses 》, Genre en séries [En ligne], 11 | 2020, mis en ligne le 01 juin 2020, consulté le 18 février 2021. URL http://journals.openedition.org/ges/1067 ; DOI : https://doi.org/10.4000/ges.1067

Ce document a été généré automatiquement le 18 février 2021.

\section{(i) $\odot$

La revue Genre en séries est mise à disposition selon les termes de la Licence Creative Commons Attribution - Pas d'Utilisation Commerciale - Pas de Modification 4.0 International. 


\section{L'espion au complet gris :} masculinité et performance dans $L a$ Mort aux trousses

Traduction de «The Spy in the Gray Flannel Suit », in Steven cohan, Masked Men : Masculinity and the Movies in the Fifties, Bloomington and Indianapolis, Indiana University Press, 1997, p. 1-33.

\section{Steven Cohan}

Traduction : Jules Sandeau

Je remercie Liam Costigan, Geneviève Sellier, Létitia Mouze et Steven Cohan pour leur aide (NdT). 


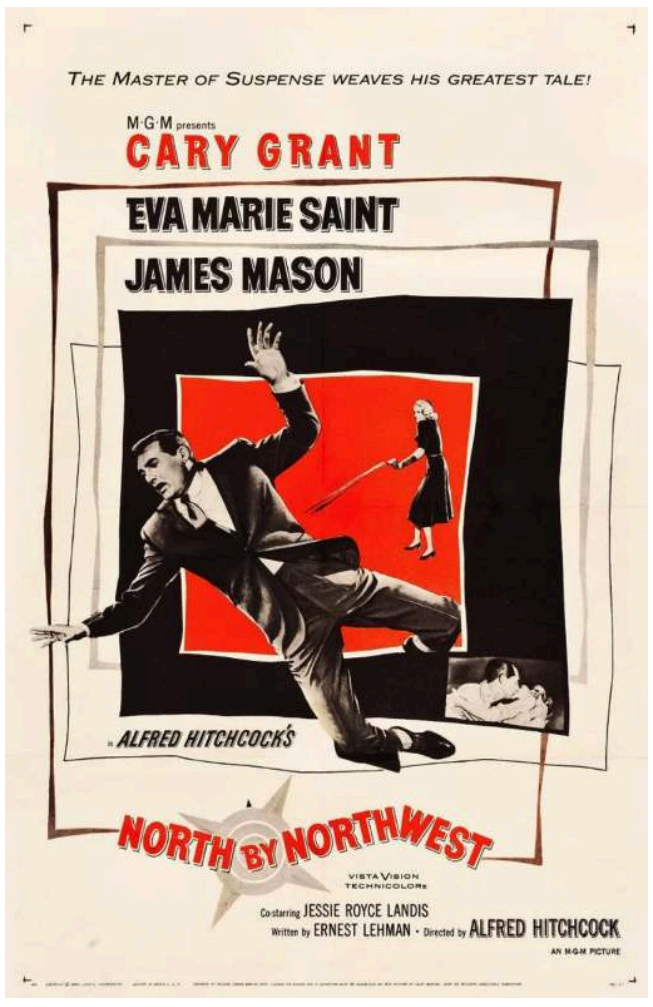

(North by Northwest, 1959)

1 L'une des affiches originales de La Mort aux trousses (North by Northwest, 1959) offre une image saisissante de vulnérabilité masculine : Cary Grant suspendu en l'air, impuissant. Bien qu'il étende ses bras comme s'il s'apprêtait à amortir sa chute avec ses mains, ses jambes pliées et la position de ses pieds indiquent une totale incapacité à contrôler son corps quand il heurtera le sol. Qui plus est, l'expression qui se lit sur son visage bouche grande ouverte, yeux fermés - témoigne de sa peur, voire de sa terreur face à cette situation désespérée. La présence d'Eva Marie Saint à l'arrière-plan, derrière Grant, avec un revolver à la main - un dispositif qui donne l'impression qu'elle est la cause de la situation dangereuse dans laquelle il se trouve - nous encourage à y voir une référence à un passage du film : quand Eve Kendall (Saint) fait semblant de tirer sur Roger O. Thornhill (Grant) - qui se fait passer pour l'espion américain fictif, George Kaplan -, afin de convaincre son amant, Phillip Vandamm (James Mason), que son attachement et sa loyauté envers lui n'ont pas faibli. Le choix de cette scène pour l'affiche était bien sûr calculé de la part du studio. Les personnes qui n'avaient pas encore vu le film interpréteraient ce geste d'Eve comme le fait Vandamm, en déduisant qu'Eva Marie Saint joue le rôle d'une sirène perfide qui met en péril la vie de Cary Grant. La voix-off de la bande-annonce encourage le public non-initié à appréhender la relation entre Grant and Saint de la même manière. Elle commence par s'adresser à la star elle-même : « Tu n'y peux rien, Cary. Quelqu'un est lancé à ta poursuite... ». Et elle se conclut en promettant au public « de nombreux rebondissements. L'audacieux Cary, séduit par le genre de blonde qui sait comment s'y prendre pour qu'un homme l'ait dans la peau, et n'hésite pas à utiliser des balles de revolver pour arriver à cette fin ». Sur quoi la bande-annonce montre des images de la fameuse scène en guise d'illustration. 
2 Les matériaux promotionnels attirent l'attention du public sur une scène cruciale de La Mort aux trousses, qui est en fait le tournant du film, puisqu'il s'agit du moment à partir duquel Roger Thornhill devient un héros à part entière, qui agit plus qu'il ne réagit ${ }^{1}$. George M. Wilson soutient que ce faux meurtre représente «la mort de Roger Thornhill» (65), mais il serait plus exact de dire qu'il symbolise la mort de George Kaplan, l'identité fictive qui a servi de persona ${ }^{2}$ masculine à Roger. Après ce simulacre de meurtre, Roger prend en charge l'histoire pour la première fois. Désobéissant aux ordres du Professeur (Leo G. Carroll), il change de vêtements et s'échappe de l'hôpital, où « George Kaplan » a été mis en lieu sûr, pour aller rejoindre Eve en son nom propre et de son propre chef, signalant à cette dernière qu'il est caché dans la maison de Vandamm en jetant devant elle la boîte d'allumettes sur laquelle figurent ses initiales (« R. O. T. »). Inutile de dire que, si l'assassinat de « Kaplan » par Eve n'était rien d'autre qu'une mascarade, la masculinité héroïque que Roger exhibe lorsqu'il vole ensuite à son secours, est présentée comme authentique.

3 Tandis que l'affiche donne l'impression que le corps de Cary Grant a été projeté en l'air par les coups de feu d'Eva Marie Saint, lorsque Roger simule sa chute dans le film, il tombe au sol immédiatement après qu'Eve lui a tiré dessus, et atterrit avec une force telle qu'il se fait un bleu sur le flanc, qu'il montre au Professeur dans la chambre d'hôpital. Peut-être que l'image de la star en l'air, impuissante, est mise en avant sur l'affiche parce que le climax du film sur le mont Rushmore - quand Eve perd l'équilibre et tombe avant d'être rattrapée par Roger - joue sur le même type de danger que celui de la conclusion de Sueurs froides (Vertigo, 1958), le film qu'Hitchcock a tourné juste avant La Mort aux trousses. Plus probablement, dans la mesure où La Mort aux trousses ne se finit pas sur une mort tragique comme celle qui concluait le film précédent (Cavell : 775), le poster évoque la scène de chute qui ouvre Sueurs froides, dans laquelle Scottie (James Stewart), à la poursuite d'un criminel, perd l'équilibre et s'accroche au toit d'un immeuble. Sueurs froides tiendra ensuite des discours contradictoires sur la vulnérabilité terrifiante de Scottie sur le toit, en expliquant d'une part que sa peur du vide résulte de son trauma qui continue de le hanter sous la forme de vertiges récurrents, tout en le présentant d'autre part comme une faiblesse congénitale qui précédait son trauma et a été la cause de sa paralysie lorsqu'il a perdu l'équilibre. «Ce n'était pas le moment idéal pour découvrir que je souffrais de ça ", déclare Scottie à Midge (Barbara Bel Geddes) à propos de sa peur du vide. La Mort aux trousses suscite cette même peur de la chute, avec les mêmes connotations d'émasculation, et sa conclusion, comme celle de Sueurs froides, soulage cette angoisse en la déplaçant sur une figure de femme plus vulnérable ${ }^{3}$. Durant ce climax, l'équilibre de Roger est garanti par des appuis relativement fermes sur le monument, alors qu'Eve est suspendue en dessous de lui, impuissante, et n'est préservée d'une chute mortelle (dont est victime Judy/Kim Novak dans Sueurs froides) que par la main du héros - ce qui pousse le sbire sadique de Vandamm, Leonard (Martin Landau), à écraser l'autre main de Roger avec son talon. L'affiche, au contraire, enlève le sol sous le corps de Cary Grant, sans lui laisser aucun appui, de la même manière que la scène introductive de Sueurs froides abandonnait Scottie sur le toit, confronté à une situation d'émasculation à la fois circonstancielle et innée.

4 Bien qu'une image de vulnérabilité puisse sembler incompatible avec le pouvoir qu'incarnent habituellement les stars hollywoodiennes masculines pendant la période du système des studios, elle est hautement appropriée dans le cas de la campagne 
promotionnelle orchestrée par la MGM pour La Mort aux trousses. Le département de publicité du studio est même allé jusqu'à reproduire l'affiche au début de la bandeannonce. En réintroduisant du danger dans la fausse chute du héros, pour ainsi dire, l'affiche exploite la même peur d'être émasculé par une femme armée et dangereuse que la conclusion de La Mort aux trousses s'efforce d'exorciser - et même plutôt de réduire à néant puisque, dans la diégèse, elle tire sur lui avec des balles à blanc. Telle que l'affiche la dépeint, la «chute » de Cary Grant est une image assez terrifiante de masculinité en crise, et le film lui-même ne peut pas totalement exorciser cette angoisse même si sa conclusion sur le mont Rushmore en donne l'impression.

Je souhaite examiner comment La Mort aux trousses, à l'image de sa campagne promotionnelle, met en scène une masculinité en crise. L'énorme succès public et critique du film lors de sa sortie en juillet 1959 est dû sans nul doute à son mélange d'aventure et de comédie, pas à sa pertinence sociale. Loué comme le retour d'Hitchcock au sommet après les échecs commerciaux du Faux coupable (The Wrong Man, 1956) et de Sueurs froides, La Mort aux trousses apparaît encore aujourd'hui comme « le film hitchcockien par essence » (Kapsis 56) : un divertissement pour adultes, palpitant et romantique, qui semble avoir très peu de conscience historique, n'être aucunement préoccupé de problèmes sociaux réels, et qui, contrairement à Fenêtre sur cour (Rear Window, 1954) ou Sueurs froides, ne dépeint pas comme problématique la masculinité valorisée au sein d'une culture patriarcale. Cependant, le fait que le climax final prenne place sur le mont Rushmore indique clairement que ce film est loin d'être idéologiquement innocent, ni déconnecté de son contexte historique. Quelles que soient les intentions d'Hitchcock et du scénariste Ernest Lehman lorsqu'ils ont créé "Roger O. Thornhill», ce personnage était une intuition commercialement perspicace de ce que les États-Unis des années 1950 voulaient penser, pour le meilleur et pour le pire, de la masculinité dans ce contexte.

\section{Le mariage fait l'homme}

Depuis l'ouvrage auteuriste de 1965 dans lequel Robin Wood étudie le savoir-faire avec lequel Hitchcock embarque Roger Thornhill dans une aventure palpitante s'achevant par son union avec Eve Kendall, le conservatisme social et sexuel de La Mort aux trousses a été démontré à plusieurs reprises. À travers ses confrontations successives aux trois visages de l'Eve du film (d'abord séductrice, puis traitresse, et finalement partenaire du héros), Roger est guéri de sa dépendance vis-à-vis de sa mère, de sa méfiance envers les femmes et de sa peur de l'engagement ${ }^{4}$. Il est ainsi tentant d'adhérer à la thèse de Leslie Brill qui voit dans le film une quête mythique à travers laquelle «Roger Thornhill se cherche une identité et une partenaire appropriée - deux facettes d'un même but ici » (8). Cependant, l'enjeu central du film est bien plus historiquement déterminé que ça, dans la mesure où il partage la croyance culturelle dans le fait que « le mariage - et, à l'intérieur de ce cadre conjugal, le fait de rapporter l'argent à la maison - est le seul état normal pour l'homme adulte" (Ehrenreich, 1983:15). La Mort aux trousses associe ainsi explicitement l'accès de son héros à la maturité au fait de se marier. S'il veut revivifier la masculinité condescendante, hypocrite, manipulatrice et égocentrique qu'il exhibe dans la première scène sur Madison Avenue, Roger doit apprendre la différence entre « des avances» ( a proposition»), terme qu'il emploie pour décrire les intentions d'Eve lorsque qu'elle lui confesse avoir soudoyé le serveur pour le faire 
assoir à sa table dans le wagon-restaurant, et « une demande " («a proposal»), terme qu'il utilise, lorsqu'ils sont encore sur le monument, pour l'inviter à prendre le même train pour rentrer à New York.

Dans La Mort aux trousses, comme dans beaucoup d'autres films des années 1950, l'éthique du breadwinner ${ }^{5}$ donne lieu à une trajectoire narrative apparemment simple qui aboutit au mariage et à l'accès à la maturité du héros. Mais avant cette conclusion, le comportement de Roger parodie en fait cet idéal pendant la majeure partie du film. Lorsque le Professeur lui demande de l'accompagner à Rapid City pour neutraliser les soupçons de Vandamm vis-à-vis d'Eve, il lui répond: "Je suis un publicitaire, pas un leurre! J'ai un travail, une secrétaire, une mère, deux ex-femmes, et plusieurs barmen qui comptent sur moi. Je les décevrais quelque peu en me faisant tuer». Si la formulation insiste sur les personnes qui dépendent économiquement de lui dans sa vie privée, la déclaration désinvolte de Roger indique en réalité son irresponsabilité. La manière dont le psychiatre Abram Kardiner diagnostique le "momisme" résume la conception de la masculinité pendant les années 1950 d'une manière qui décrit parfaitement Roger à ce moment-là :

L'attachement à une mère forte et dominatrice peut avoir différentes conséquences sur le caractère. Prenons ainsi le cas d'un homme de 35 ans, fils unique, qui se plaint de rencontrer des difficultés avec les femmes. Aucun trouble de l'activité sexuelle, seulement une difficulté à s'adapter aux femmes. Il a eu beaucoup d'aventures relativement dénuées de tendresse. Ses difficultés proviennent de sa volonté de prendre aux femmes sans donner en retour... [Sa mère] le protégeait, mais de manière tyrannique. Il recherche maintenant une vie dans laquelle il peut reproduire sa relation avec sa mère, afin d'être l'objet exclusif de l'attention de son épouse et d'être soutenu par elle. Si les femmes n'étaient pas aujourd'hui aussi disponibles pour des «aventures", il ne les fréquenterait pas du tout (Kardiner : 28).

8 En attribuant à Roger ce type de comportement envers les femmes, au nombre desquelles sa mère capricieuse, le film encourage le public à voir en lui un exemple de "l'immaturité émotionnelle croissante de l'homme américain ", un syndrome touchant les soldats revenus du front et qui s'enracine, selon un expert, dans l'incapacité de beaucoup d'hommes à s'adapter à un environnement militaire pendant la Seconde Guerre mondiale. "La plupart d'entre eux étaient des hommes émasculés ", soutenait ce psychiatre. «Ils voulaient dépendre de quelqu'un d'autre. Au lieu de pourvoir et de protéger, ils voulaient être protégés. Ils n'avaient jamais appris à accepter la responsabilité - ils avaient perdu de vue l'idéal masculin » (cité dans Coughlan : 114). Si l'immaturité de Roger semble confirmer la thèse psychiatrique répandue d'une interruption du développement masculin, elle évoque autant l'idéologie de la guerre froide que l'éthique du breadwinner. La manière dont le personnage est caractérisé confirme l'hypothèse selon laquelle les déficiences émotionnelles de l'homme américain (indiquées par les divorces et les problèmes d'alcool de Roger), qui sont la cause de son irresponsabilité sociale et personnelle (comme le révèle le discours de Roger au Professeur), l'ont rendu excessivement dépendant d'une femme forte (sa mère) et ainsi exposé à des influences néfastes (le communisme).

Comme je l'ai évoqué dans l'introduction, l'impression que les hommes américains séduisants de classe moyenne comme Roger ont «perdu de vue l'idéal masculin » et sont devenus « des hommes émasculés » participait d'un " déclin de la confiance et du prestige national » qui dominait la fin de la décennie (Oakley: 413). L'affolement concernant les modèles de masculinité atteignit un tel degré que le magazine Look 
publia en 1958 une série d'articles analysant ce qu'il appelait «le déclin de l'homme américain ${ }^{6}$ ». Le premier article donnait le ton de cette enquête: "Les scientifiques s'inquiètent du fait que, depuis la fin de la Seconde Guerre mondiale, l'homme américain a changé radicalement et dangereusement; qu'il n'est plus l'homme masculin et déterminé qui a conquis le continent et fait la grandeur de l'Amérique " (Moskin : 77). Chaque article de la série se concentrait sur un des symptômes de la crise de la masculinité qui touchait la nation : le fait que les hommes se laissaient dominer par les femmes, le fait qu'ils travaillaient trop dur au péril de leur santé physique et spirituelle, et le fait qu'ils se conformaient beaucoup trop facilement aux valeurs communes. Avec son titre faisant allusion à Gibbon ${ }^{7}$ et au motif de la chute de l'empire, cette série d'articles cherchait clairement à agiter le spectre d'une perte de pouvoir des États-Unis dans le domaine de la politique internationale :

Malgré des conséquences néfastes sur son système nerveux, l'homme américain a acquis pour lui et sa famille des biens et des services sans équivalent dans les autres pays du monde. (...) (Le danger, bien sûr, est que nous devenions trop mous, trop paresseux et trop centrés sur le foyer pour relever le défi posé par d'autres nations dynamiques comme la Chine et l'Union Soviétique.) (...) La solution - si elle existe est que l'homme américain grandisse émotionnellement pour être capable de supporter la pression de cette société et de répondre aux besoins de son entreprise, de sa communauté et de son foyer sans ruiner sa santé et son tempérament. (Attwood : 74-75).

10 La Mort aux trousses fait plusieurs références ponctuelles au contexte de guerre froide dans lequel prend place la réhabilitation de Roger Thornhill par son mariage avec Eve Kendall. Le Professeur remarque par exemple, après la scène du faux meurtre, que les États-Unis ont déjà perdu quelques guerres froides, et craint manifestement qu'ils subissent une nouvelle défaite. Dans la mesure où cette idée est explicitée par l'inquiétude du Professeur vis-à-vis de la perte de puissance des États-Unis face aux communistes, le public de 1959 a sans doute interprété le portrait initial de Roger en fils à maman séducteur et alcoolique, et sa transformation finale en homme responsable guéri par le mariage, comme une représentation du déclin et du renouveau de l'homme américain. Dans sa manière d'assimiler la masculinité à la nation, cette représentation était typique de l'époque :

La logique était la suivante. La puissance nationale dépendait de la capacité d'hommes forts et virils à lutter contre la menace communiste. Ce n'était pas seulement une question de faiblesse générale conduisant à une politique étrangère molle; bien plutôt, la décadence ou les excès sexuels feraient des individus des proies faciles pour les stratégies communistes. Selon l'idéologie dominante de l'époque, le comportement hétérosexuel "normal » culminant dans le mariage, représentait la « maturité » et la « responsabilité »; par conséquent, les « déviants » qui ne correspondaient pas à cette norme étaient, par définition, irresponsables, immatures et faibles. Il s'ensuivait que les hommes qui étaient esclaves de leurs passions, pouvaient être facilement dupés par des femmes séductrices travaillant pour les communistes. Encore pires étaient les «pervers» qui étaient vraisemblablement dépourvus de toute détermination masculine (May : 94).

11 Ce qui est particulièrement remarquable à propos de la paranoïa culturelle décrite ici par Elaine Tyler May, est la manière dont de telles représentations de l'intérêt national imaginaient si ouvertement les divergences politiques entre l'Union soviétique et les États-Unis en termes genrés, en présentant la guerre froide comme un conflit entre masculinité et féminité, avec la sexualité perverse servant de trope dominant pour représenter ce qui était perçu comme des déséquilibres de pouvoir. 

est déréglée que Roger peut être dupé par une femme fatale (nommée Eve, rien de moins !) travaillant pour les communistes. Tout aussi important, l'association du communisme à l'émasculation et à la perversion (une manière homophobe d'imaginer l'ennemi redouté comme une inversion du breadwinner masculin et de tout ce qu'il incarne dans la société américaine) explique le fait que Vandamm soit efféminé, un trait signifié par ses manières britanniques réservées et hautaines, ses goûts de luxe et son intérêt pour les antiquités, ainsi que sa relation intime avec un sadique homosexuel, son secrétaire-compagnon, Leonard. C'est peut-être une coïncidence, mais comment interpréter le fait que la crise identitaire de Roger commence lorsqu'il est " chopé $^{8}$ " par les hommes de Vandamm dans la Oak Room du Plaza Hotel ? Selon George Chauncey, il s'agissait d'un « lieu de rendez-vous bien connu chez les hommes gays (...), où les hommes étaient censés bien s'habiller et se comporter avec discrétion » (350). Quoiqu'il en soit, lorsque Roger met en échec Vandamm l'efféminé en fuyant avec Eve et la figurine contenant les microfilms volés, son triomphe équivaut à une revitalisation masculine et nationale.

13 Tandis que la fin du film confirme que Roger est devenu un homme digne de ce nom grâce à sa rédemption en tant qu'amant et héros, reste qu'il ne vainc pas Vandamm et Leonard tout seul mais a besoin de l'aide du Professeur - qui arrive à la dernière minute avec un garde forestier, lequel utilise cette fois des vraies balles. Loin de diminuer ou de saper le statut acquis par Roger à la fin du film, l'intervention à point nommé du Professeur semble curieusement renforcer ce statut. Parce que Roger a agi de son propre chef quand il a quitté la chambre d'hôpital pour aller chercher Eve, l'aide finale du Professeur le replace sous l'autorité de l'État en soulignant la convergence de leurs intérêts. Michael Rogin fait remarquer que les récits de guerre froide se terminent généralement par la victoire de l'homme libre qui triomphe des agents communistes tout en restant, paradoxalement, dépendant de « la puissance armée de l'État ». Ce type de conclusion a pour effet de révéler la similarité des moyens par lesquels les ÉtatsUnis et leurs ennemis communistes assoient leur pouvoir étatique (militarisme, espionnage, surveillance). Afin d'échapper à cette contradiction idéologique, une fausse antinomie "entre maternité et communisme » semble résoudre la contradiction en augmentant d'un cran cette paranoïa culturelle qu'était la "peur des rouges ", en assimilant l'absorption par l'empire communiste à un engloutissement dans la féminité (Rogin : 240). C'est exactement de cette manière que procède la conclusion de La Mort aux trousses sur le mont Rushmore, en assimilant les deux forces potentiellement les plus perturbatrices et écrasantes, qui doivent donc être contenues par le pouvoir masculin : le communisme à l'extérieur et la sexualité féminine à l'intérieur. Comme le soutient Robert J. Corber, en soulignant que La Mort aux trousses est le film d'Hitchcock des années 1950 qui reproduit le plus franchement le consensus de l'après-guerre sur la politique de guerre froide, à partir du moment où l'histoire d'espionnage et l'intrigue amoureuse convergent pour aligner l'épanouissement de la masculinité de Roger sur l'accomplissement de son devoir patriotique, « le genre et la nationalité [fonctionnent] comme des catégories identitaires qui se renforcent mutuellement " (Corber : 191). Le destin de la nation semble ainsi dépendre de la force de la masculinité américaine, et vice versa. Cela signifie cependant que La Mort aux trousses ne peut mettre en scène cette régénération finale qu'en désignant la féminité, et non la masculinité, comme le lieu principal de la perturbation de genre au sein du récit, et pour cette raison, le happy end n'est pas aussi dénué de tensions que le soutient Corber. 


\section{La signification du « compagnonnage »} menacée caractéristique du contexte de la guerre froide, c'est son intrigue amoureuse qui lui apporte une solution : l'accès de Roger à la maturité à travers son engagement envers une femme. Avec ce déplacement vers la sphère personnelle, le film redéfinit la relation sexuelle entre Roger et Eve à travers une idéologie de l'endiguement domestique, le conflit politique de la guerre froide se jouant ainsi dans la maison de banlieue. Le terme d'« endiguement ", utilisé pour la première fois par George Kennan en 1947 pour définir une politique étrangère états-unienne efficace vis-à-vis du bloc soviétique, dominait également la vision américaine de la sécurité intérieure ${ }^{9}$, selon laquelle « un foyer rempli d'enfants génère un sentiment de chaleur et de sécurité face aux forces froides de la perturbation et de l'aliénation» (May: 23). Le foyer était supposé offrir aux couples mariés une protection contre le monde extérieur hostile, de la même manière que la nation elle-même protégeait ses citoyens contre l'empire communiste. Avec la maison individuelle célébrée comme le lieu « où presque tous les besoins personnels de ses habitants seront satisfaits par une vie privée placée sous le signe de dynamisme et de la communication » (May : 11), cette attente prit une forme concrète dans le développement des banlieues pavillonnaires, ainsi que dans les appareils électroménagers et les voitures qui symbolisaient la réussite financière du breadwinner (Clark, Spigel). En renforçant l'idéologie domestique de la guerre froide, l'obsession nationale pour le couple hétérosexuel contribua à contenir la sexualité masculine et féminine à l'intérieur d'une forme d'union relativement nouvelle, «le mariage érotisé » (May: 133-134). En tant que seul lieu légitime de satisfaction des désirs affectifs et sexuels, cette représentation hautement idéalisée du mariage - le compagnonnage amoureux où l'unité du couple se réalise dans l'orgasme mutuel - était un instrument efficace de régulation de la sexualité. s'y attend le moins : quand Roger croit avoir été piégé par Eve. Dans la chambre d'Eve à l'Ambassador East Hotel, après la séquence où il a été attaqué par l'avion, Roger lui déclare clairement qu'il ne la lâchera plus d'une semelle dorénavant, sans doute parce qu'il suppose qu'elle le mènera à son ennemi qu'il n'a jamais vu et dont il ignore le nom. "Ne serait-ce pas merveilleux si mes problèmes et tes projets étaient connectés d'une manière ou d'une autre?", lui demande-t-il. "Ainsi nous pourrions rester ensemble à partir de maintenant, sans avoir à prendre des directions différentes. Le compagnonnage. Tu vois ce que je veux dire? " Totalement étranger à cet univers d'espions et de femmes fatales, le terme de «compagnonnage (togetherness)» est apparu pour la première fois dans un numéro de McCall's de 1954 (Oakley : 119). Faisant référence aux couples mariés passant du temps ensemble et partageant équitablement les tâches domestiques réparties jusqu'ici en fonction du genre (par ex. les femmes qui font la vaisselle vs les hommes qui tondent le gazon), l'idée de « compagnonnage » était centrale dans l'idéologie domestique de la guerre froide. Elle impliquait un mari présent à la maison, prenant sérieusement ses responsabilités dans l'éducation des enfants et considérant son épouse comme la première de ses ami.e.s.

Quand Roger parle à Eve de " compagnonnage ", il le fait ironiquement puisqu'il pense alors qu'elle «pourrait allumer un homme à mort sans trop d'efforts ». Il choisit 
soigneusement ses mots pour lui signifier qu'il a compris son petit jeu et pour se venger de son apparente trahison. Cependant, l'ambiguïté des taquineries de Roger suggère aussi que ses plaisanteries défensives doivent être prises au pied de la lettre, comme un aveu sincère des sentiments croissants qu'il éprouve pour Eve. Le script d'Ernest Lehman explicite ce sous-texte en précisant que, lorsque Roger tient son discours sur le " compagnonnage », Eve " remarque, en le regardant furtivement, un sourire tendre, voire amoureux, sur son visage " (Lehman: 81). En regardant cette scène, la plupart d'entre nous ont probablement perçu plus d'hostilité et d'amertume que de tendresse et d'affection dans le ton de Roger, mais sa toute dernière réplique dans le film aide à réévaluer cette impression dans un sens plus proche du commentaire de Lehman. Tandis qu'ils s'enlacent sur la couchette supérieure de leur compartiment de train, Eve dit : «c'est idiot ", à quoi Roger répond : "Je sais. Mais je suis sentimental ». Le geste sentimental par lequel il rejoue les débuts de leur relation restaure en effet ce qu'il avait tourné en dérision plus tôt dans la chambre d'hôtel, à savoir la signification du " compagnonnage » comme nom de code pour la régulation de la sexualité au sein du couple.

Avec son insistance sur la vie de famille comme partenariat, le "compagnonnage " justifiait la division genrée que l'idéologie domestique cherchait à perpétuer (l'homme qui ramène l'argent, la femme au foyer). Roger doit donc devenir sentimental avec Eve à la toute fin du film pour que sa sexualité à elle soit contenue par le mariage, comme fondement de sa sexualité à lui. Pour reprendre les termes du film, Roger lui fait « une demande» («a proposal») parce que c'est le seul moyen de neutraliser l'initiative qu'elle prend dans le domaine de la sexualité en lui faisant «des avances» («a proposition»). Car bien qu'elle se présente à Roger en expliquant qu'elle est une célibataire de 26 ans - «Maintenant vous savez tout »-, il y a en réalité beaucoup plus à savoir sur Eve Kandall qui, si on y réfléchit, n'est pas juste une espionne mais une femme célibataire qui travaille et dont la vie sexuelle est active et non conventionnelle. Tandis qu'Eve tend à être de plus en plus passive face à tous les hommes (le Professeur, Vandamm et Roger) à partir de la scène du faux meurtre, ce n'est pas le cas au début du film, où elle sait tout de George Kaplan et de Vandamm alors que Roger ne sait rien. La «demande» que Roger fait à Eve sur le Mont Rushmore («si on s'en sort, rentrons ensemble en train à New York») revivifie sa masculinité défaillante en ramenant la femme à la maison, où il pourra contenir plus facilement les effets inquiétants de cette sexualité féminine active liés au pouvoir que possédait Eve au début du film.

Mais comment Eve, une femme, s'est-elle retrouvée dans cette position de pouvoir où elle accomplit un travail d'homme pour une agence de renseignement américaine? Quand Roger lui demande après le faux meurtre, elle répond: "c'était peut-être la première fois que quelqu'un me demandait de faire quelque chose d'utile ».

Roger : Votre vie a été comme ça ? Pourquoi?

Eve : À cause d'hommes comme vous.

Roger: Quel est le problème des hommes comme moi?

Eve : Ils ne croient pas au mariage.

Roger : J'ai été marié deux fois.

Eve : C'est bien ce que je dis...

Quand ils ont relu les premières versions du script, les censeurs de la Production Code Administration semblaient partager l'avis d'Eve. Préoccupés par l'allusion aux deux exfemmes de Roger, ils incitèrent le studio à supprimer ce détail du dialogue à deux reprises $^{10}$. Les censeurs étaient également préoccupés par la représentation de la 
sexualité d'Eve. Ils voulaient que soit inséré un dialogue établissant qu'elle n'avait jamais été la maîtresse de Vandamm (une demande qui n'a manifestement pas été prise en compte); et ils ont aussi insisté, avec plus de succès, pour que la fin suggère le mariage plus qu'« une relation sexuelle illicite » en montrant Roger et Eve « habillé.e.s pour la journée et pas pour la nuit », de sorte que rien ne suggère la consommation de leur union, une demande qui a obligé Hitchcock à tourner et à utiliser, dans la version finale du film, un plan large dans la scène finale du train (Leff :112-113).

La Mort aux trousses réduit le pouvoir d'Eve en tant qu'espionne et au sein du récit en confinant toutes ses activités futures au lit conjugal. Comme l'explicite le dialogue cité plus haut, si Roger doit croire au mariage pour être réhabilité, il a besoin du bon type de partenaire pour dépasser ses deux échecs précédents. C'est pourquoi le film transforme Eve la vamp en salope puis en Girl Scout. Une fois que le film révèle qu'Eve ne cherche ni à aider ni à piéger Roger mais qu'elle est un agent double, elle représente dès lors à la fois la cause et le remède de la crise de la masculinité. Elle a joué au soldat de la guerre froide à la place de Roger, alors que ce dernier était plus compétent qu'elle en la matière, comme le montre le fait qu'il découvre le moyen par lequel Vandamm fait sortir les microfilms du pays (dans des antiquités), un secret qu'elle n'avait pas réussi à percer. Cette situation de crise traversée par Roger, dont le point de départ était une simple erreur d'identité, se révèle avoir de gros enjeux politiques: Roger montre aux agents du gouvernement américain comment déjouer l'ennemi et gagne une bataille de la guerre froide, faisant ainsi preuve de la masculinité «dure » que requièrent les affaires étrangères; et il réhabilite les vertus ternies du breadwinner (honnêteté, engagement et fidélité) à travers son mariage avec Eve, faisant ainsi preuve de la masculinité «douce» que requiert la gestion des affaires domestiques. Parce qu'Eve est la figure assurant l'articulation de l'intrigue amoureuse et de l'histoire d'espionnage, la fin du film l'utilise pour éviter de reconnaître que ces deux constructions très différentes de la masculinité sont en fait incompatibles.

\section{Attention à la blonde}

Dissimulant ses véritables intentions derrière un visage inexpressif et des paroles provocantes, Eve Kendall semble une incarnation parfaite de la blonde hitchcockienne. Sa personnalité dégage cette impression de sexualité "glaciale » dont Grace Kelly est l'incarnation emblématique selon le réalisateur, avec son sex-appeal "indirect» reposant sur «le paradoxe du feu qui brûle sous la glace", pour reprendre les termes de François Truffaut (224). Ce type spécifique de sexualité glaciale est un ingrédient essentiel de ce que Sabrina Barton a judicieusement nommé « la fonction de la blonde » dans le cinéma d'Hitchcock (Barton: 76). En tant que femme dont le corps attire le regard du spectateur, la blonde hitchcockienne est l'incarnation du spectacle féminin au cinéma, la blondeur rayonnante de son image fétichisée offrant au public une projection du désir masculin autant que la blancheur chatoyante de l'écran de cinéma. Cette manière intéressante de conceptualiser l'effet de la blonde hitchcockienne trouve son illustration la plus parfaite dans Vertigo, selon Barton, avec la transformation de Judy la brune en Madeline la blonde platine.

22 Pourquoi la blondeur a-t-elle cet effet spectaculaire sur la masculinité, particulièrement dans la manière dont l'exploite Hitchcock ? Ce n'est pas un hasard si, à l'exception de Madeleine Carroll dans ses films britanniques des années 1930, ses 
actrices blondes appartiennent toutes aux années 1950 : Grace Kelly, Doris Day, Vera Miles, Kim Novak, Eva Marie Saint, Janet Leigh. Ses stars les plus célèbres des années 1940, Joan Fontaine et Ingrid Bergman, avaient certes les cheveux clairs, mais n'étaient pas identifiées et présentées comme des blondes (à la manière de Betty Grable par exemple). Comme il l'explique à Truffaut à propos de son choix d'actrices blondes comme Grace Kelly: "Nous cherchons des femmes du monde, de vraies dames qui deviendront des putains dans la chambre à coucher. La pauvre Marilyn Monroe avait le sexe affiché sur le visage, et Brigitte Bardot n'était pas non plus très subtile" (Truffaut : 224). De la même manière qu'il y a toujours eu des stars féminines blondes, il existe aussi une opposition traditionnelle entre héroïnes lumineuses et héroïnes sombres dans la mythologie sexuelle occidentale, comme dans les romans historiques de Sir Walter Scott du début du $19^{\text {ème }}$ ou dans les films noirs des années 1940. Mais en opposant deux types de stars blondes, celles qui ont le sexe affiché sur le visage et celles qui ne l'ont pas, la remarque d'Hitchcock aide à replacer la blondeur, quelle que soit son type, dans le contexte historique des années 1950 où elle constituait la métaphore principale de la sexualité féminine. Pendant cette période, les stars féminines devinrent de plus en plus blondes tandis que leurs homologues masculins restaient "grands, bruns et séduisants ", pour reprendre la célèbre formule ${ }^{11}$.

Représentant la féminité la plus désirable, la blondeur est évidemment le signe de la suprématie de la masculinité blanche. C'est ce que signifiait le "sexe » affiché sur le visage (et même sur le corps entier) de Monroe, pour reprendre les termes dans lesquels Hitchcock fait allusion à la blonde la plus célèbre des années 1950. Richard Dyer souligne que, « pour devenir la femme idéale, Monroe devait être blanche. Et pas seulement blanche mais blonde, c'est-à-dire la plus blanche possible» (Dyer, 1986b : 42-43). Il note également que, dans le cas de Monroe, la blondeur associait sa féminité à une position sociale connotant richesse et supériorité raciale. Suite à la victoire militaire des États-Unis et à leur occupation de l'Allemagne nazie (promouvant ellemême une idéologie de la blondeur masculine) et du Japon au terme d'une guerre traversée par le racisme blanc, la préférence pour les femmes blondes pendant les années 1950 était le signe de la supériorité des hommes américains qui les possédaient. La mode de la blondeur pendant cette période (au cinéma comme dans la société) reflétait la valorisation croissante de l'identité WASP par une nation qui commençait à voir la suprématie blanche remise en question à l'intérieur du territoire (avec le boycott des bus de Montgomery, qui initia le mouvement des droits civiques en 1955 et cristallisa les peurs liées au blockbusting sporadique qui commençait à se développer dans les quartiers blancs ${ }^{12}$ ) comme à l'extérieur (avec l'enlisement croissant des ÉtatsUnis dans des conflits incertains impliquant des nations non-blanches et nouvellement indépendantes d'Asie et d'Afrique).

Est-ce que le fait que la blancheur extrême de certaines blondes hollywoodiennes des années 1950 soit aussi surdéterminée par les codes culturels de l'époque ne la rend pas étrange, en particulier pour le public d'aujourd'hui à qui elle apparaît d'autant plus datée et artificielle? Pauline Kael va jusqu'à affirmer que le look d'Eva Marie Saint dans La Mort aux trousses transforme son visage en "masque africain albinos» (Kael : 418). Même s'il ne s'agit pas là d'un compliment, l'image évoquée n'est pas si farfelue qu'elle en a l'air, dans la mesure où la couleur de cheveux et le maquillage de l'actrice, accentués par l'éclairage lumineux et les costumes sombres, connotent le caractère non naturel du blanc, le fait que cette couleur signifie l'absence, comme sur un tissu blanchi ou un visage qui pâlit. Comme le souligne Dyer, «quand la blanchité est regardée en 
tant que blanchité, elle apparaît souvent comme un vide, une absence, un déni ou même une sorte de mort » (Dyer, $1988: 44)$. C'est bien sûr ce blanc qui terrifie et fascine en même temps Ishmael dans Moby Dick - et c'est aussi, à mon avis, la raison pour laquelle les femmes fatales des années 1950, comme Eve Kendall, tendent à être blondes.

Malgré sa fonction idéologique apparemment stable, la blondeur s'avère un symbole extrêmement instable du patriarcat blanc. D'un côté, le look artificiel des blondes des années 1950, qu'Hitchcock justifie à travers la dichotomie "dames »/« putain », est, comme en témoigne sa comparaison, la forme ultime de déni des différences sexuelles, sociales et raciales. D'un autre côté, si la blondeur, et tout particulièrement sa version platine si populaire pendant les années 1950, est, comme le dit Dyer, la blanchité la plus parfaite qu'une femme peut atteindre, c'est aussi pour beaucoup de femmes la plus artificielle. Peu de femmes américaines pouvaient passer pour des blondes platine naturelles, et peu le voulaient. Paradoxalement, plus la blonde apparaît comme une construction artificielle de la beauté féminine, plus elle naturalise avec succès la suprématie économique et raciale de l'homme blanc qu'elle est conçue pour séduire.

Ce paradoxe est lié au fait que la blonde acquiert son statut culturel en attirant une attention excessive sur la fabrication de la blanchité féminine en tant que moyen principal de dénier le fait que le patriarcat blanc se fonde lui-même sur un système symbolique; tandis que, en même temps, l'attention attirée sur sa blondeur met en péril ce qu'elle symbolise, conduisant ainsi potentiellement l'ensemble de ce système de représentation au bord de l'effondrement. Trop d'attention portée à la blanchité en tant que construction symbolique peut révéler que l'inscription de la blondeur sur le corps des femmes est une contrefaçon, comme dans Vertigo ; ou, à l'autre extrême, cela peut diluer la figure féminine dans la blondeur à un point tel qu'elle évoque alors la surface vierge ou vide connotée par la couleur blanche, un effet qui, s'il n'est pas récupéré sous la forme du stéréotype de la blonde idiote incarné par Monroe dans ses films, se matérialise sous la forme de la femme fatale: "le genre de blonde qui sait comment s'y prendre pour qu'un homme l'ait dans la peau, et n'hésite pas à utiliser des balles de revolver pour arriver à cette fin ". Significativement, dans ce slogan, la blonde est impénétrable alors que l'homme ne l'est pas. Avec sa blondeur qui joue le rôle d'une façade empêchant le regard masculin de la percer à jour, «son sexe " n'est pas affiché sur son visage, qui refuse tout simplement de jouer sa fonction spéculaire ${ }^{13}$, présentant au regard masculin « un masque africain albinos» - c'est effectivement la meilleure manière de le dire - qui suggère comment la blondeur peut brouiller l'opposition clair/ sombre qu'elle sert à construire.

La fonction symbolique de la blondeur explique pourquoi Eve Kendall reste impossible à cerner pendant la majeure partie du film, contrairement aux hommes, qui s'avèrent avoir des visages plus facilement déchiffrables. Non seulement les visages familiers de présidents constituant le décor des scènes finales évoquent la présence rassurante $d u$ patriarcat, mais le visage de Roger est lui-même instantanément reconnaissable dès le moment où il rencontre Eve dans le train :

Roger: Je sais, ma tête vous semble vaguement familière.

Eve: Oui.

Roger: Vous avez l'impression de m'avoir déjà vu quelque part.

Eve: Oui.

Roger : Ça m'arrive tout le temps. Ça tient à mon visage.

Eve : C'est un beau visage. 
tinuant dans la même veine, ce dialogue est ironique dans la mesure où Eve réussit facilement à lire le visage de Roger tout en le dupant au sujet de sa propre identité et de ses intentions. Quand il déclare que «les femmes honnêtes [1]'effraient " parce qu'« elles [le] mettent dans une situation désavantageuse », Eve suppose que c'est parce qu'il n'est pas « honnête avec elles », témoignant ainsi de sa capacité à le cerner avec rapidité et précision. Son commentaire pointe un défaut de Roger, mais il indique aussi à quel point il est incapable de cerner les femmes; elle badine ici avec lui avec un sérieux qu'il ne détecte pas. Malgré son autosatisfaction, Roger n'a vraiment aucune idée de ce qu'Eve cherche à faire, à savoir lui montrer qu'il a effectivement encore beaucoup à apprendre sur le fait d'être mis dans une situation désavantageuse par une femme intelligente.

Plusieurs critiques ont noté que les agissements secrets d'Eve dans le récit de La Mort aux trousses révèlent une gamme d'émotions beaucoup plus grande que ce qu'elle laisse apparaître, puisqu'elle est assez attirée par Roger pour coucher avec lui dans le train, assez impitoyable pour l'envoyer se faire tuer le lendemain matin, assez émotive pour laisser paraître son soulagement quand il survit, et assez attachée à lui pour être blessée par ses insultes lorsqu'il réapparaît inopinément à la vente aux enchères (Wood: 139; Wilson: 75). Cependant, la focalisation du film sur Roger renvoie la subjectivité d'Eve à la marge de l'action principale, de sorte que le drame au cœur de son histoire à elle reste hors-écran. Cette position accentue encore plus l'ambiguïté de sa personnalité puisqu'elle implique que ses intentions ne peuvent être comprises qu'indirectement, une chose rendue encore plus difficile par la maîtrise de soi dont elle fait preuve. Quand je disais plus haut qu'Eve avait trois visages qui se succèdent à travers une série de transformations apparentes, j'ai un peu exagéré, en me référant à l'impression que le dénouement du film nous donne, semblant gérer si parfaitement le mystère de ce personnage. Il est plus juste de dire que ses trois visages ne cessent d'interagir dans le film, ce qui est la source de la menace qu'elle représentante pour la masculinité en tant que blonde.

Cette complexité fait d'Eve un personnage très différent du méchant du film l'organisation secrète qui assigne une nouvelle identité à Roger - dans la mesure où Hitchcock a, comme il l'explique à Truffaut, décidé avec Lehman de «diviser ce méchant en trois personnages : James Mason, séduisant et suave ; son secrétaire à l'air sinistre ; et le troisième espion, frustre et brutal » (Truffaut : 107). Significativement, ces hommes sont tous bruns, tandis que le personnage qui résiste à la division, Eve, est blonde, un trait qui ne reflète pas la bonté morale, mais l'opacité résultant de sa capacité à changer de visage. Par son contraste avec la clarté produite par la division du méchant en personnalités distinctes, l'ambiguïté produite par la mascarade d'Eve marque la différence sexuelle dans le film, avec la transparence des hommes bruns opposée à l'impénétrabilité de la femme blonde. La scène du faux meurtre représente un tournant dans la relation de Roger à la féminité d'Eve, puisque son pouvoir de femme impossible à cerner est contrôlé au sein d'une mascarade masculine: la performance de Roger subordonne ainsi l'activité d'Eve (en tant que personnage actif du récit) à la sienne, maîtrisant ainsi la sexualité féminine dans le cadre de sa propre mascarade en tant que George Kaplan. Mais c'est aussi précisément la raison pour laquelle Eve cause tant d'ennuis à Roger en tant que blonde. Car lorsque, en accomplissant sa "fonction de blonde", elle offre une image en miroir de sa 
performativité à lui, Eve finit par révéler ce que la blonde est supposée masquer : le fait que la masculinité blanche repose elle aussi sur une mascarade de genre.

\section{C'est un publicitaire, pas un leurre}

31 Même s'il est important de comprendre comment différents aspects du contexte idéologique de la guerre froide (l'éthique du breadwinner, le conflit politique global, l'endiguement domestique et la blanchité) s'entremêlent dans la résolution de La Mort aux trousses, appréhender la masculinité de Roger uniquement sous ce prisme fait passer ce film pour beaucoup plus sombre et sérieux que ne le justifient son scénario et sa mise en scène. Après tout, avec ses dialogues pleins d'esprit, La Mort aux trousses nous fait rire autant qu'il nous tient en haleine. Avec une ironie souvent dévastatrice, tandis qu'il s'achemine vers sa résolution conservatrice sur le Mont Rushmore où Roger fait sa demande à Eve et sauve la nation des ennemis communistes, le film ne cesse de placer les discours dominants de l'Amérique des années 1950 dans des contextes très déplacés, avec pour effet de de rendre insolites des concepts familiers tels que " compagnonnage », qui était déjà passé dans le langage commun en 1959. La séquence où Roger est attaqué par l'avion fonctionne de la même manière, en rendant sinistre un banal champ de maïs de l'Indiana, de la même manière que les nombreuses blagues du film mobilisent des clichés à des moments inopportuns, comme lorsque l'imperturbable Mrs. Thornhill (Jessie Royce Landis) demande à son fils quarantenaire, alors que celui-ci est en train de fuir les hommes de Vandamm au Plaza Hotel : « Roger, seras-tu rentré à la maison pour l'heure du dîner?"

Les gags rejouent verbalement ou visuellement ce que le film lui-même fait à Roger en le dépossédant de son identité lorsque les hommes de Vandamm le prennent pour Kaplan. Cette méprise n'est pas due à une quelconque ressemblance physique mais à une association accidentelle de Roger au nom de Kaplan. Non seulement Roger est pris pour "George Kaplan», qui n'existe qu'à travers un ensemble de traces (des métonymies de la masculinité : une brosse à cheveux, des costumes, un nom) laissées par l'équipe du Professeur pour convaincre Vandamm de l'existence de ce leurre, mais en cherchant à prouver son innocence, Roger découvre comment les signes conventionnels de sa propre masculinité (sa relation aux affaires, à l'alcool, aux femmes et son complexe d'ÆEdipe - tous évoqués dans la conversation entre Roger et sa secrétaire au début du film) peuvent être facilement dissociés de son identité très banale. Comme j'ai commencé à le sous-entendre dans mes remarques sur la blondeur d'Eve, l'identité de Roger est, en dernière analyse, une persona ou un masque semblable à l'identité fabriquée de Kaplan. C'est seulement lorsqu'il prend conscience de cela que Roger commence à prendre le dessus sur ses adversaires en les battant à leur propre jeu : la performance.

\section{Les habits font l'homme}

Dans un livre important republié la même année que celle de la sortie du film, le sociologue Erving Goffman soutenait que la performance était en fait la base de l'identité, «la présentation de soi dans la vie quotidienne ». Publiée originellement en 1956 sous la forme d'une monographie académique plus courte, cette analyse des diverses formes d'interactions professionnelles et personnelles comme des exemples de 
"gestion de l'impression (impression management)» décrit bien les jeux de rôle qui interviennent à maintes reprises dans la diégèse de La Mort aux trousses. En faisant dire à Roger : « les publicitaires ne mentent jamais, ils exagèrent juste la vérité de manière avantageuse ", le film commence déjà à le rapprocher de Vandamm et du Professeur. Goffman montre que cet axiome ne s'applique pas uniquement aux mondes de la publicité ou de l'espionnage, mais caractérise les relations sociales en général : « Dans la vie quotidienne, il est généralement possible pour l'acteur de susciter intentionnellement presque n'importe quel type de fausse impression sans avoir à mentir franchement. Des techniques de communication comme le sous-entendu, l'ambiguïté volontaire, et les omissions cruciales permettent au désinformateur de tirer profit du mensonge sans en dire aucun au sens strict du terme » (Goffman : 62).

Dans La Mort aux trousses, la scène du faux meurtre semble marquer la fin de ce type de jeu de rôle en faisant tomber le rideau sur les performances qui avaient jusqu'ici fait avancer l'action. Après cela, Vandamm découvre la véritable identité de l'agent double (Eve), Roger va la sauver en son propre nom, le Professeur intervient avec des vraies balles, et toutes les tromperies et simulations nécessaires à l'espionnage semblent bien exclues de la vie privée à laquelle retournent Mr. et Mrs. Thornhill. Mais le film développe en fait un propos plus complexe sur les jeux de rôle des espions de la Guerre froide. Ainsi, tout en s'évertuant en apparence à conforter la représentation la plus conventionnelle de la masculinité, comme l'ont soutenu les critiques de Wood à Corber, La Mort aux trousses reconnaît aussi, avec autant de conviction, le fait que ce type de représentation sociale s'enracine dans des performances, ce qui enlève à l'identité la stabilité que lui confère l'idéologie patriarcale-nationaliste pour la faire apparaître au contraire comme une production sociale dynamique et instable qui requiert le déploiement perpétuel de masques, de jeux de rôle, et de théâtralité ${ }^{14}$.

Dans la mesure où le film avance que toutes les identités ne peuvent se réaliser qu'à travers des performances de ce que Goffman appelle une « activité signifiante » (2), on peut se demander ce qui fait qu'une identité (celle de Roger disons) a plus de réalité qu'une autre (celle de Kaplan). Je suppose que la réponse est: celle qui nous va le mieux. Pendant tout le temps où la foi de Roger en son identité est remise en cause, et où l'assurance que le public qui regarde le film pour la première fois a de l'innocence de Roger peut même être ébranlée lorsque les hommes de Vandamm le prennent pour George Kaplan, il y a un élément qui reste constant, fixe et invariable tout au long de ses nombreuses proclamations sincères d'innocence et de ses performances accidentelles de culpabilité. Je veux parler du complet gris apparemment indestructible que Roger porte quasiment tout le temps jusqu'au faux meurtre, après quoi son changement de vêtements indique qu'il prend enfin en charge le déroulement du récit.

Avant qu'il délaisse son costume en même temps que sa persona de George Kaplan, Roger reste d'une élégance irréprochable. Ses vêtements semblent propres et fraîchement repassés en toutes circonstances, à la fois pour suggérer l'indestructibilité du héros, semblable à celle d'un personnage de dessin animé, et pour renvoyer à l'image de Cary Grant qui était connu pour son sens de la mode et ses goûts précis en matière de vêtements. Selon un communiqué de presse de l'époque :

Ses chemises, toujours sur mesure, possèdent un rabat dissimulant les boutons. Il ne plie jamais ses mouchoirs de poche en triangle, mais toujours en carré et les range négligemment. Ses cravates ne sont jamais pointues, mais toujours arrondies. Il préfère les cravates monochromes, ou une cravate de couleur sombre avec des petits motifs parsemés. Il ne porte jamais de cravates claires, et plus généralement 
aucune couleur claire sur ses vêtements.

Son costume de ville favori est gris foncé, à rayures, droit, et il porte toujours une chemise crème. Il préfère une cravate grise, d'une nuance plus sombre ou plus claire que son costume. Avec cela, il porte des chaussettes en soie et laine, parfois unies, parfois avec une ligne de motifs verticale. Il préfère les chaussures très foncées, d'une couleur presque bronze ${ }^{15}$.

Comme le suggère également cette description de la garde-robe de Grant, le complet gris sur mesure et apparemment inaltérable que porte le héros de La Mort aux trousses est également un marqueur de classe instantanément reconnaissable. «Le héros est un publicitaire (un choix de profession significatif) », note Lawrence Alloway au moment de la sortie du film en 1959, « et bien qu'il soit pourchassé de New York au Dakota du Sud, ses vêtements restent impeccables, tout droit sortis de chez Brooks Brothers. Autrement dit, la saleté, la sueur et les dégâts causés par la poursuite sont moins importants que l'emballage dans lequel est livré le héros, celui de l'homme urbain de Madison Avenue » (Alloway). Dans sa critique du film, Daily Variety voit aussi Roger comme un «homme de Madison Avenue, élégant, ayant divorcé deux fois avec insouciance, et aussi débonnaire qu'une pub pour cigarettes" (Powe). La Mort aux trousses célèbre cet emballage de masculinité à travers un de ses gags récurrents sur le charisme de Grant, notamment quand le costume de Roger devrait être froissé, comme lorsqu'Eve le range dans son petit sac de voyage pour qu'il puisse sortir du train déguisé en porteur, ou après la séquence de l'avion, quand il décide de le faire laver et repasser, pour qu'Eve ne se doute pas qu'il la suit et le conduise ainsi jusqu'à Vandamm. L'un des slogans promotionnels joue sur le potentiel comique de l'indestructibilité des vêtements de Roger: «Les espions surgissent de partout... fuyez les policiers, les tueurs, les jolies femmes... et voyez si vous pouvez faire tout ça sans froisser votre costume! » (pressbook).

Pourquoi le costume de Roger est-il si important qu'il doive être - et soit effectivement - mentionné ${ }^{16}$ ? Il est révélateur que le scénario publié dépeigne son costume d'une manière légèrement différente du souvenir que l'on en garde généralement: «[Roger est] grand, mince, impeccablement habillé (et bien trop original pour porter le même uniforme gris que ses semblables)» (Lehman : 2). Ce n'est pas faux, d'autant plus que la couleur du costume gris que porte Cary Grant pendant la majeure partie du film varie en fonction de la lumière et du décor, de sorte qu'il semble parfois plus bleu que gris, et à d'autres moments plus gris que bleu. Mais même si le costume de Roger n'est peut-être pas l'uniforme en flanelle que tout le monde va acheter chez Brooks Brothers - a en effet été taillé sur mesure pour Grant chez Quintino de Wilshire Boulevard (Higham et Moseley : 273) - pendant presque tout le film (jusqu'à la scène du faux meurtre), les personnages qui entourent Roger portent des vêtements plus sombres (gris foncé, bleu marine, noir) pour accentuer la couleur grise de ses habits. En d'autres termes, Roger personnifie visuellement cette contradiction savoureuse du conformisme des années 1950 que Lehman décrit dans son scénario. Dans son costume de marque, Roger Thornhill est instantanément reconnaissable comme un représentant « original » de son milieu, les cadres de classe moyenne, dont les valeurs sont arrivées à dominer la culture de l'après-guerre.

L'affiliation de Roger à ce segment particulier de la classe moyenne est ce qui le caractérise de la manière la plus saillante, la plus stable et la plus incontestable. Seul élément majeur de continuité pour lui tout au long du film, cette identité reste assez claire pour investir son personnage d'une autorité sociale, si ce n'est morale, quoiqu'il 
fasse et en toutes circonstances. Comme La Mort aux trousses ne cesse de placer son héros en complet gris devant un public, cela a pour effet de conférer une autre signification politique à sa masculinité, moins évidente mais tout aussi pertinente historiquement que celle que lui confèrent les différentes idéologies de la Guerre froide motivant la résolution du film. L'importance de «l'homme au complet gris » en tant que figure omniprésente dans la culture de l'époque est due en grande partie à la manière dont la publicité, de plus en plus dirigée autant vers les hommes que vers les femmes, repensa une dichotomie genrée supposée alors fondamentale: production masculine vs consommation féminine. Le lancement des magazines Playboy et Sports Illustrated en 1953 témoigne d'une reconnaissance du marché grandissant et prometteur que constituent les consommateurs masculins, comme la naissance des industries "do it yourself». "L'homme au complet gris» était perçu comme l'incarnation de cette absorption de la masculinité au sein du consumérisme, qui allait à l'encontre des représentations de genre conventionnelles mais dont tout le monde reconnaissait également le potentiel économique. De plus, dans la mesure où il était perçu à la fois comme un producteur et un produit de l'industrie de la publicité, il en vint à personnifier la fusion de la production et de la consommation.

En tant que cadre d'une entreprise de publicité, Roger Thornhill est l'illustration de la transformation qui s'est opérée pendant l'après-guerre, du publicitaire débraillé et peu recommandable - incarné notamment par Clark Gable dans Marchands d'illusions (The Hucksters, 1947) - au publicitaire respectable et bien habillé. « Vous êtes un publicitaire, c'est tout ce que ce je sais ", dit Eve à Roger dans le train entre deux baisers. "Vous avez du goût en matière de vêtements. Du goût en matière de cuisine (...). Vous maniez habilement les mots. Vous pouvez probablement leur faire faire tout ce que vous voulez. Vendre à des gens des choses dont ils n'ont pas besoin. Faire tomber amoureuses de vous des femmes qui ne vous connaissent pas ». Même si sa rhétorique peut donner l'impression qu'elle a lu La Persuasion clandestine (The Hidden Persuaders) de Vance Packards, Eve dit tout cela sur un ton qui témoigne plus de son excitation que de sa désapprobation. Malgré la critique de la masculinité de Roger sous-entendue par son association aux publicitaires de Madison Avenue, il n'empêche qu'il suscite une grande sympathie chez le public comme chez Eve, dès le début du film où sa vie est mise en danger. Son charme est renforcé par la performance décontractée et pleine d'assurance de Cary Grant, mais aussi par l'insistance du film - en dépit de l'immaturité et l'insincérité de Roger, sans parler de sa ressemblance avec les espions en tant qu'expert en masques et performances - sur le fait qu'il s'agit de quelqu'un d'ordinaire, un homme d'affaires stressé qui se retrouve plongé dans l'univers trouble des complots et des services secrets. À vrai dire, les spectateurs/trices ordinaires n'ont pas le pouvoir économique et la position sociale que Roger considère comme allant de soi dans la scène introductive sur Madison Avenue; mais il n'empêche que le film encourage le public à s'identifier à Roger en tant que «Monsieur Tout-le-monde de la ville moderne ", pour reprendre l'expression de Robin Wood (134) - tout particulièrement à partir du moment où son arrogance et sa malhonnêteté commencent à être corrigées.

41 Si l'on voulait être plus précis, cependant, il serait plus juste de décrire Roger non pas comme un homme ordinaire, mais comme le type d'homme le plus emblématique des années 1950. Sa profession l'associe directement à une force sociale émergeante dans les États-Unis de l'après-guerre, que l'historien Jackson Lears décrit comme : « un bloc hégémonique (...) formé de groupes souvent décrits comme une "nouvelle classe" de managers, administrateurs, universitaires, techniciens et journalistes - des gens qui ne 
fabriquent pas des choses mais manipulent plutôt des symboles, et dont les compétences sont organisationnelles, techniques, conceptuelles ou langagières " (Lears : 50). Analysés pendant cette période à travers les concepts d'« individu extrodéterminé $^{17}$ " (David Reisman), d'« homme d'organisation ${ }^{18}$ " (William H. Whyte) et d'« élite diplômée " (Vance Packard), ces cadres ayant fait des études supérieures étaient, pour reprendre les mots de Packard, « les gens importants et actifs qui avaient réussi et qui avaient globalement le pouvoir ", par opposition aux « classes subalternes comprenant les cols bleus et cols blancs lambda passifs : les petits commerçants, les ouvriers, fonctionnaires, assistants» (41). Comme l'observait Packard en 1959, la convergence récente entre l'« authentique classe supérieure" (celle qui a hérité du capital) et la " classe semi-supérieure » (celle des chefs d'entreprise et des cadres ayant fait des études supérieures) a divisé la classe moyenne apparemment homogène, avec des conséquences considérables sur la société états-unienne. Du fait de ce «brouillage inédit de la frontière entre les cols blancs et cols bleus » (36), les membres de la classe moyenne inférieure subissaient les conséquences d'une réorganisation historique des affiliations sociales dont ils n'eurent pas conscience, continuant à identifier leurs intérêts économiques et politiques à ceux de leurs employeurs sans percevoir le fossé de classe qui les séparaient.

Techniquement, le nouveau groupe constitué par ces cadres de la supervision et des médias n'avait pas la cohérence et l'unité d'une «classe » au sens strict du terme, mais il possédait cependant un pouvoir réel et une influence palpable en tant que «bloc hégémonique (...) identifiant ses propres problèmes et intérêts avec ceux de la société, et même de l'humanité tout entière ». Comme le souligne Lears, il s'agissait d'une " coalition de groupes qui différaient sur beaucoup de points mais qui étaient liés (dans une certaine mesure) par des intérêts communs, des expériences communes et une vision du monde commune " (Lears 51). Bien qu'elle commença ensuite à se diviser pendant les années 1960 au sujet des droits civiques et de la guerre du Vietnam, certain.e.s historien.ne.s désignent cette coalition et ses transformations successives au cours des années 1970 et 1980, sous le terme de "classe moyenne professionnelle » afin de donner un nom à ce groupe social particulier qui (1) se distingue par un «statut économique et social basé sur des études plutôt que sur la possession de capitaux ou de biens », et (2) « utilise la consommation pour établir son statut, tout particulièrement par rapport aux classes populaires » (Ehrenreich, 1989 : 12-14).

Même à l'époque de sa formation dans l'immédiat après-guerre, cette nouvelle classe de cadres-managers était déjà perçue comme reflétant le passage des États-Unis d'une économie organisée autour de la production à une économie de plus en plus déterminée par la consommation. Par exemple, dans un article de 1954 répondant à la question "Qu'est-ce que l'homme américain?", le Time attirait l'attention sur les conséquences de ce tournant radical dans l'économie états-unienne et appréhendait ensuite la nation à travers le point de vue hégémonique de ces hommes qui ont fait de la consommation leur business :

De manière croissante, les entreprises se regroupent en conglomérats et les hommes d'affaires se tournent vers leurs concurrents, plutôt que vers leur propre département de comptabilité, pour mesurer leur succès. Leur attitude vis-à-vis de leur propre travail n'est pas celui de producteurs mais de consommateurs. La confiance est au plus haut quand une décision reçoit l'approbation (et suscite l'imitation) des « collaborateurs concurrents » du groupe de pairs. (Anon. 1957 : 23) 
44

observations figuraient dans un gros article portant sur La foule solitaire (The Lonely Crowd) de David Reisman, publié quatre ans plus tôt. En 1954, ce livre extrêmement influent avait déjà popularisé l'expression commune servant à décrire la personnalité du nouvel homme d'affaires de classe moyenne tourné vers la consommation: le conformiste "extro-déterminé ", incarnation de l'homme d'entreprise du $\mathrm{XX}^{\mathrm{e}}$ siècle, qui est un consommateur, par opposition à l'individualiste "intro-déterminé ", incarnation du capitaine d'industrie du XIX ${ }^{\mathrm{e}}$ siècle, qui était un producteur. À la suite de Reisman, le Time décrit comment l'économie consumériste de l'après-guerre a transformé la mentalité de l'homme d'affaires au point de la remodeler autour de l'échange de ce que Goffman allait bientôt analyser comme des signes de performance : pour réussir en affaires, les compétences communicationnelles requises exigeaient une sensibilité aux apparences extérieures et compréhension de l'identité sociale comme persona ou masque. Au milieu de la décennie, aucun cadre ne symbolisait mieux l'utilisation du capital dans l'unique but de stimuler la consommation que « l'homme au complet gris ", le publicitaire dont le travail était littéralement la manipulation de signes et qui se fondait sans aucune difficulté dans la foule des hommes d'affaires anonymes et semblables qui remplissaient les rues de tous les centres villes américains - ce que La Mort aux trousses évoque d'ailleurs dans son plan introductif de Madison Avenue. En isolant Roger de cette foule, le film dramatise ainsi immédiatement sa capacité à "signaler son succès " parce qu'il montre comment ce cadre se prend rapidement au jeu de la performance en réaction à ses propres «collaborateurs concurrents ", Vandamm et ses hommes.

À partir du moment où Vandamm prépare involontairement le terrain à Roger pour que celui-ci pense son identité comme une performance, cet espion en complet gris se révèle être l'incarnation parfaite du cadre "extro-déterminé » et de l'idéologie de la Guerre froide qui conforte l'hégémonie de sa classe en réaffirmant la valeur de sa masculinité. Dans la première scène, La Mort aux trousses satirise gentiment Roger en tant que cadre typique de Madison Avenue; dans la deuxième, le film remet brutalement en question son identité ; et à partir de ce moment, le film cherche à restaurer son affiliation avec la classe des cadres-managers afin de légitimer l'hégémonie culturelle de cette dernière au sein de la société américaine. En sauvant à la fois la femme qu'il aime et les secrets qui avaient été volés à l'État, Roger vit l'histoire d'amour de l'aventurier de salon de l'après-guerre. L'urgence de cette idylle, comme le suggère le film en affiliant Roger à la nouvelle classe des cadres-managers, résulte du besoin de valider sa masculinité qui est plus menacée par son travail que par Vandamm. "Les vocations "viriles" ou masculines typiques sont en train de disparaître rapidement", remarque le psychiatre Henrik M. Ruitenbeek: "Si les occupations masculines sont de moins en moins importantes dans une économie technologique - et de surcroît orientée essentiellement vers la finance et les relations publiques plutôt que vers la production - le rôle masculin dans la famille a aussi changé » (86). Événement pour le moins surdéterminé idéologiquement, la victoire de Roger à la fin de La Mort aux trousses cherche à restaurer la masculinité menacée du cadre de classe moyenne.

\section{Performer les genres}

Parce que la fin du film semble se consacrer avec tant de zèle à aligner la masculinité revitalisée de Roger et l'intérêt national, il est important de comprendre que La Mort 
aux trousses dramatise aussi la manière dont l'éthique de la performance de la classe des cadres-managers pourrait facilement défaire ce lien entre idéaux de genre et nationalisme. La persona "extro-déterminée " de cette nouvelle classe minait sévèrement la stabilité et la singularité de l'«identité » en lui substituant un ensemble varié de types sociaux, ce que le sociologue Orrin E. Klapp décrit comme une construction identitaire "de style cafétéria " (98) dans un livre publié au début des années 1960. Portant un regard rétrospectif sur la société états-unienne des années 1950, Klapp remarquait que, contrairement à ce que soutient l'idéologie individualiste américaine, «nous n'avons pas, dans notre société, une liberté par rapport aux types, comme on pourrait le penser au premier abord, mais un choix entre des types» (2). Significativement, il caractérisait la diversité et la confusion de la mise en scène de soi dans la société américaine grâce à une métaphore assimilant la performance de l'identité à l'économie consumériste qui la sous-tend : "Les Américain.e.s se trouvent dans une sorte de magasin de vêtements soldés, avec un nombre déconcertant [de rôles] entre lesquels choisir - les modèles ne nous vont peut-être pas tous aussi bien, mais rien ne nous empêche de les essayer » (96).

Roger est l'incarnation parfaite de ce que Klapp décrit, en infléchissant le concept de personnalité "extro-déterminée » de Riesman, comme «le héros d'aujourd'hui, déterminé par son public (audience-directed), [qui] peut jouer tous les rôles avec aisance, de l'homme du monde au pilier d'église. Il est partout chez lui, a "une présence scénique", et parvient toujours à retomber sur ses pieds " (107). La maîtrise de la performance dont témoigne Roger, qui lui permet de survivre aux situations dangereuses auxquelles il est confronté au cours de son périple du Nord-Est des ÉtatsUnis jusqu'à l'arrière-pays du Midwest, redéfinit sa personnalité comme un lieu de changements identitaires répétés. Ces derniers ont lieu successivement, à travers un ensemble de contextes idéologiques proches mais pas identiques, et chaque performance est une mise en scène au sein de laquelle sa personnalité adopte une persona masculine différente (le cadre de Madison Avenue, la victime de kidnapping, l'alcoolique, le fils à sa maman, le meurtrier, le fugitif, l'amant, le porteur, etc.). C'est aussi vrai pour Eve. À chaque fois qu'elle réajuste son masque pour s'adapter au scénario particulier du moment, elle révèle en même temps qu'elle ne possède pas une identité stable qui transcenderait les circonstances l'obligeant à adopter telle ou telle persona féminine pour s'en sortir. Cette blonde est dangereuse, non parce qu'elle a un pistolet, mais parce qu'elle tire à blanc de bien des manières. Elle illustre le fait que toute identité sociale est, pour reprendre encore une fois l'expression de Goffman, une "activité signifiante" nécessitant des aptitudes théâtrales, une flexibilité dans le maniement de ses multiples personae, et une attention aux exigences d'un public en perpétuelle évolution. Comme la masculinité de Roger, sa féminité n'est pas expressive, au sens où elle renverrait à une identité stable en-deçà des couches de mascarades (la « vraie » Eve), mais elle est performative, au sens où elle se reconstitue en permanence dans une représentation théâtralisée.

En montrant comment l'acte social de mise en scène de soi déstabilise la continuité de l'identité personnelle, La Mort aux trousses met en question l'authenticité de toute représentation. La classe des cadres-managers tirait sa position sociale dominante et une bonne partie de ses revenus du contrôle des représentations, mais elle se méfiait aussi par principe des représentations. Les scandales qui ont touché le monde de l'espionnage puis celui des jeux télévisés pendant cette période ont attisé les 
appréhensions concernant les dangers de l'instrumentalisation professionnelle des représentations. Dans ce contexte, les publicitaires et leur travail ont été l'objet de critiques répétées à l'intérieur même de la nouvelle classe. Jouant le rôle de boucs émissaires de l'hypocrisie et du conformisme dominant le monde de l'entreprise, l'industrie de la publicité et ses hommes en complets gris étaient accusés d'assimiler la substance à son emballage - et même suspectés, comme dans Un pyjama pour deux (Lover Come Back, 1961), de faire pire que ça: vendre un emballage vide à un public ne se doutant de rien. Tel qu'imaginé par La Mort aux trousses, le gouvernement américain luimême adopte cette attitude extrêmement cynique en inventant George Kaplan. Même si « Kaplan » n'est rien de plus qu'un ensemble de signes genrés, Roger et Vandamm sont persuadés de son existence pendant la majeure partie du film. Toutefois, Hitchcock et Lehman informent très tôt le public du caractère fictif de Kaplan afin de souligner qu'une représentation convaincante, loin de devoir nécessairement se référer à ce qui en est à l'origine dans la réalité, se substitue en fait à sa source - au point de simuler facilement son propre fondement.

L'instabilité de la représentation dans La Mort aux trousses, a des conséquences particulièrement perturbatrices sur la stabilité apparente de la masculinité dans ses divers contextes idéologiques. Car si toute identité sociale est caractérisée par la substitution d'une persona à la " vraie " personne, alors pourquoi les genres, qui sont un des aspects de l'identité, ne seraient pas eux aussi de l'ordre de l'imitation et du faux ? En posant cette question, j'ai à l'esprit les travaux de Judith Butler théorisant le genre comme une performance continuelle, "la stylisation répétée du corps, un ensemble d'actes répétés à l'intérieur d'un cadre normatif très rigide, qui se cristallisent avec le temps pour produire l'apparence d'une substance, de quelque chose de naturel » (Butler, 1990 : 33). Elle explique ensuite :

La distinction entre expression et performativité est cruciale. Si les attributs et les actes genrés, les diverses manières dont un corps exhibe ou produit sa signification sociale, sont performatifs, alors il n'y a pas d'identité préexistante à l'aune de laquelle un acte ou un attribut pourrait être évalué ; il ne peut pas y avoir des actes de genre vrais ou faux, authentiques ou déformés, et le postulat d'une véritable identité de genre se révèle être une fiction régulatrice. (1990 : 141)

De ce point de vue, la « masculinité » ne renvoie pas à une nature masculine mais imite plutôt une "fiction régulatrice » normative qui autorise la représentation continuelle de certains types de performances de genre pour les hommes (comme celle du breadwinner), en marginalise d'autres (comme celle du fils à sa maman), et en interdit d'autres (comme l'homosexuel efféminé). De même, Butler n'élabore pas son argument sur le genre sur les fictions régulatrices de la masculinité et de la féminité hétéros, mais sur leur subversion: le drag gay et lesbien. «La reproduction des constructions hétérosexuelles dans des cadres non-hétérosexuels met en relief le caractère hautement construit du soi-disant original hétérosexuel. Par conséquent, le rapport de l'homosexuel.le à l'hétérosexuel.le n'est pas celui d'une copie à un original, mais plutôt celui d'une copie à une copie ${ }^{19}{ }^{\prime}(1990: 31)$. Le genre est une représentation symbolique culturellement perçue comme mimétique ${ }^{20}$; il implique toujours un élément de mascarade, raison pour laquelle le drag, avec ses références transgressives et parodiques à la binarité de genre, dévoile si bien la fiction régulatrice des normes genrées hétérosexuelles selon Butler.

51 Certes, pendant les années 1950, les journalistes et universitaires qui critiquaient la masculinité de «l'homme au complet gris » visaient directement son comportement (sa 
passivité face aux femmes, son conformisme zélé, son investissement excessif dans le travail au péril de sa santé), pas son identité d'homme. La crise de la masculinité que Look proclame en titrant "Le déclin de l'homme américain", n'était en aucun cas présentée comme une instabilité dans, ou une perturbation de la catégorie de "genre » elle-même. Cependant, le cadre "extro-déterminé " était tout de même identifié de manière genrée - il était, après tout, un homme en complet gris. La théorisation butlerienne du genre comme performance aide à formuler ce qui n'était pas affirmé directement pendant les années 1950 mais continuellement suggéré par l'anxiété de cette période à propos d'une crise de la masculinité. Par exemple, dans un autre de ses articles sur la masculinité, le magazine Look reconnaît que, dans la mesure où « la masculinité est ce que la société attend de l'homme qu'il fasse et qu'il soit » (Frank 53, les italiques sont dans l'original), alors l'éducation continue des hommes à la " masculinité » signifie aussi qu'être un homme n'est pas nécessairement la même chose que faire ce qu'un homme doit faire. "Le genre est toujours un faire ", affirme Butler, « mais ce n'est pas un faire accompli par un sujet qui lui préexisterait» (1990:25). Si la masculinité était effectivement performative, comme le sous-entendait Look, alors il s'agissait d'une fiction socialement déterminée, une représentation d'attributs masculins sans origine en deçà de l'acte de représentation lui-même. La performance de genre nécessite que les hommes et les femmes s'engagent dans des mascarades semblables à celles de Roger et Eve dans La Mort aux trousses, accomplissant ce que la culture attend des hommes et des femmes qui souhaitent apparaitre respectivement « masculins » ou « féminines » de manière appropriée.

Dans les études féministes sur le cinéma et les études culturelles, il existe maintenant toute une tradition d'analyse de la féminité comme mascarade dans l'esprit de la célèbre étude de cas publiée par Joan Riviere en 1929, qui ramenait la féminité à la mascarade et posait que ce masque dissimulait un vol féminin du phallus. Selon elle, " la féminité peut par conséquent être endossée et portée comme un masque, à la fois pour cacher la possession de la masculinité et pour éviter les représailles qui menacent les femmes si cette possession de la masculinité est découverte». La conclusion de Riviere, selon laquelle il n'y a aucune différence entre "la féminité authentique et sa “mascarade" » - « une telle différence, radicale ou superficielle, n'existe pas » (38) -, a dominé les analyses de la mascarade féminine au cinéma, particulièrement lorsqu'il est question de l'activité spectatorielle et de la subjectivité féminines ${ }^{21}$. Le concept de mascarade a récemment été appliquée également aux hommes, mais parce qu'il a été si souvent utilisé pour expliquer la construction culturelle de la féminité, on présuppose encore que la mascarade a pour conséquence une féminisation implicite de l'homme (comme dans les exemples de Riviere elle-même sur le passing des hommes gay portant le masque de la virilité hétérosexuelle) ${ }^{22}$. Même Vested Interests, l'étude impressionnante de Marjorie Garber sur la fonction culturelle du travestissement, prend presque quatre-cents pages pour conclure que l'analyse du genre en termes de mascarade conduit à reconnaître "que "l'homme" - la personne de sexe masculin - est au moins aussi artificiel que "la femme" " (Garber : 374$)$.

Mon analyse de la mascarade masculine dans La Mort aux trousses - comme dans tous les autres films étudiés dans ce livre - s'intéresse plus à sa dimension théâtrale qu'à sa dimension phallocentrique. Par conséquent, j'utilise ce terme dans l'esprit de la théorisation butlerienne du genre comme "performatif - c'est-à-dire comme produisant l'identité qu'il prétend être » (Butler, $1990: 25)$, ce qui revient à considérer les expressions d'une identité de genre à la fois comme ses causes et ses conséquences ${ }^{23}$. 
Ce type de mascarade de genre ne dissimule pas le vol du phallus, comme dans la formulation de Riviere, mais définit plutôt l'identité en opposant - et en déplaçant des plans pour donner une impression de spatialité : un rapport entre extérieur et intérieur, surface et profondeur, figure et fond, performance et authenticité. Loin de soulever des questions de dissimulation et de camouflage pour suggérer une identité cachée, plus authentique, même s'il lui manque toujours quelque chose, ce que la persona genrée - le masque lui-même - produit est le signe ou la performance de ces différences.

Cette conception de la mascarade de genre est particulièrement adaptée au cinéma dans la mesure où ce dernier repose sur les stars en tant qu'industrie. La star de cinéma - la personnalité à l'écran n'est pas nommée "persona " par hasard - amène sur le devant des représentations populaires les problèmes épistémologiques que Butler décrit dans sa déconstruction des identités de genre. D'un côté, la star entretient une relation apparemment fixe et authentique à un référent dans le monde réel, comme l'illustrent notamment les photographies utilisées abondamment par l'industrie pour promouvoir les films et leur interprètes principaux (Dyer, 1986a : 135). Mais d'un autre côté, la star est aussi une « image » dans un sens qui n'est pas purement visuel. Comme Dyer l'explique dans Stars, l'«image de star» est une production de l'industrie, une construction qui résulte d'une interaction complexe - souvent surdéterminée, parfois sous-déterminée - d'éléments biographiques, de rôles à l'écran, de gestes et de postures, et de discours médiatiques, qui s'enrichit, rarement sans contradictions, tout au long de la carrière d'un interprète. Loin d'être calquée sur la personne réelle, l'image de la star à l'écran est elle-même une copie de copie, un masque ou une persona censée authentifier un type social, racial, sexuel et genré dans le contexte théâtral d'un film et de sa promotion.

Dans leurs performances de genre, les stars hollywoodiennes produisent un effet comparable au drag dans la mesure où elles brouillent elles aussi des oppositions binaires apparemment rigides, comme être/paraitre, naturel/artificiel, sincère/ trompeur, qui recoupent elles-mêmes l'opposition masculin/féminin. Selon Dyer, « les images de star sont essentiellement liées à des contradictions au sein d'une idéologie ou entre différentes idéologies, qu'elles cherchent à "gérer" ou à résoudre de différentes manières » - ou même à subvertir dans la mesure où une image de star peut être le lieu d'une "tension entre des codes" (Dyer, 1986a: 38). Même si, comme le note Dyer, toutes les stars incarnent des "valeurs menacées", certaines stars sont extrêmement populaires à certains moments de leur carrière parce que leur persona à l'écran dramatise clairement, ou du moins provoque, une rupture idéologique (32). Le fait que Cary Grant, élu star masculine la plus populaire de 1958, puisse si bien représenter la masculinité américaine hétérosexuelle, en dit long sur l'ambiguïté et l'instabilité de la "masculinité » dans la culture de l'époque. Par conséquent, tandis que La Mort aux trousses avance et multiplie les allusions clairement réflexives à la présence de sa star dans le rôle principal, la persona de " Cary Grant » associe encore plus Roger Thornhill à la performance de genre, d'une manière qui doit être analysée sérieusement si l'on veut comprendre pleinement la signification de la crise que traverse sa masculinité dans le film. 


\section{Le visage familier de Cary Grant}

56 Même s'il était une star hollywoodienne populaire depuis les années 1930, Grant fit une sorte de comeback à la fin des années 1950 en regagnant les sommets du box-office grâce aux succès d'Elle et lui (An Affair to Remember, 1957), Indiscret (Indiscreet, 1958), Opération jupons (Operation Petticoat, 1959) et La Mort aux trousses. Contrairement à d'autres stars de sa génération (comme John Wayne, Humphrey Bogart, Gary Cooper, James Stewart), dont les rôles des années 1950 transformaient la persona en tenant compte de leur âge (Biskind : 252-53), Grant ne semblait alors aucunement affecté par le passage du temps, et avait même encore plus de glamour et de charme en tant qu' «authentique héros américain» (Rothman: 177) ou «monument national» (Cavell : 769). En 1958, quand Photoplay inclut Grant, présenté comme "l'incarnation hollywoodienne du charme romantique ", dans une série de photos de stars masculines posant dans des piscines, cela ne paraissait pas du tout étonnant - ni inapproprié - de trouver l'acteur de 54 ans aux côtés d'idoles du public féminin comme Tony Curtis, Rock Hudson, George Nader et Hugh O'Brian (Anon. 1958, 95). Et il n'était pas non plus étrange de voir, après le succès de La Mort aux trousses, le « débonnaire » Cary Grant figurer entre le « rebelle » Dwayne Hickman et le « roi du campus » Troy Donahue dans un guide pour les célibataires publié par le magazine en 1960 (Anon. 1960 : 47).

57 Malgré son statut légendaire de star masculine quintessentielle du cinéma hollywoodien des années 1950, Cary Grant était un héros romantique un peu paradoxal pour la période. Ses biographes parlent de "son masque " lorsqu'ils évoquent sa persona à l'écran pendant les années 1950, une expression qui, en faisant allusion à ses "problèmes sexuels", désigne «sa fausse image, si soigneusement entretenue, de masculinité sans équivoque et de forte stabilité émotionnelle» (Higham et Moseley: 248). Tout en incarnant avec succès, à l'écran et à la ville, un idéal de jeunesse éternelle, d'américanité, de charme hétérosexuel, de célibat et d'élégance vestimentaire, Grant était en fait un homme d'âge mûr, britannique, bisexuel, marié (il n'avait pas divorcé de Betsy Drake), et se travestissant en secret (il portait apparemment des sous-vêtements féminins en nylon sous ses costumes taillés sur mesure ${ }^{24}$ ). En résumé, sa persona d'après-guerre redéfinissait son âge, son appartenance de classe, sa nationalité, son orientation sexuelle et sa situation familiale pour lui faire incarner quelque chose de totalement différent : l'homme d'affaires américain célibataire et éternellement jeune, le rôle qu'Hitchcock et Lehman lui ont taillé sur mesure dans La Mort aux trousses (Spoto : 436).

58 À la fin des années 1950, ce «Cary Grant » fabriqué de toutes pièces passe tellement pour le parangon de la masculinité romantique américaine, que lorsque Billy Wilder demande à Tony Curtis d'imiter sa célèbre manière de parler, il peut être sûr que le public de Certains l'aiment chaud (Some Like It Hot, 1959) comprendra immédiatement cette allusion comique. "Où est-ce que tu as chopé cet accent bidon?", demande Jack Lemmon à Curtis après avoir regardé ce dernier se faire passer pour un héritier célibataire afin d'attirer l'attention de Marylin Monroe: «Personne ne parle comme ça ». Mais tou.te.s les spectateurs/trices du film de Wilder savaient qui parlait vraiment comme ça - la plupart des critiques mentionnaient d'ailleurs Cary Grant lorsqu'ils évoquaient cette blague - bien que le public de l'époque était divisé sur la question de savoir s'il s'agissait d'un hommage, d'une satire, ou juste d'un pur commérage sur la vie privée de la star. 
59 Sorti à peine quelques mois avant La Mort aux trousses, Certains l'aiment chaud offre néanmoins une lecture très fine de la persona de Cary Grant dans le contexte des années 1950. Plus qu'une simple référence à une icône culturelle de la séduction romantique, cette allusion perspicace à Grant représente sa célèbre persona comme une performance, désignée spécifiquement comme une mascarade au sein de laquelle la « masculinité » est construite par la voix, les vêtements, la posture - tout cela étant, comme le souligne le film de Wilder, emprunté à quelqu'un d'autre pour dissimuler une défaillance fondamentale de la sexualité masculine. "Je suis inoffensif », confesse Tony Curtis à Marilyn Monroe avec la voix de Cary Grant; et bien que le déguisement masculin ne vise ici qu'à dissimuler son agressivité hétérosexuelle (en feignant l'impuissance, Curtis pousse Monroe à prendre toutes les initiatives dans le rapport de séduction), la référence à Grant est une allusion à la face cachée troublante de la star. Elle sous-entend assez clairement que cette célèbre image de charme et d'élégance cache en fait l'autre grande peur de la culture américaine des années 1950 à propos de la masculinité: non pas la subversion communiste ou la guerre atomique, mais l'impuissance, culturellement assimilée à l'émasculation, une peur attisée notamment par les deux rapports Kinsey et la révélation très médiatisée du second volume selon laquelle la sexualité féminine atteignait son pic relativement tard, alors que les performances sexuelles masculines commençaient à décliner beaucoup plus tôt ${ }^{25}$.

60 Cependant, l'allusion à "Cary Grant» dans Certains l'aiment chaud va au-delà d'une simple suggestion d'imposture phallique, parce qu'elle assimile totalement la persona de l'acteur à une performance consciente de la masculinité. Loin de n'être qu'une simple blague sur la vigueur sexuelle d'une star hollywoodienne légendaire, l'allusion tente de rendre compte de son style romantique très maniéré mais toujours aussi apprécié. Quand, dans la diégèse de Certains l'aiment chaud, Tony Curtis joue un homme qui se travestit puis, tout en continuant de se déguiser en femme, se fait aussi passer pour un succédané de "Cary Grant ", l'imitation de Curtis sous-entend que Grant continue de produire cette star masculine légendaire à l'écran en manipulant des signes de performance (voix, allure, apparence) de manière similaire et tout aussi efficace. Le film de Wilder suggère ainsi que, sous cette performance accomplie de la masculinité romantique, se trouve le masque lui-même : une subversion potentielle de la manière binaire et rigide dont la culture américaine des années 1950 conçoit la masculinité en postulant une continuité naturelle, immuable et nécessaire entre le genre, la sexualité et l'anatomie. Cary Grant, ce séducteur américain débonnaire dont Certains l'aiment chaud se moque gentiment, est peut-être inoffensif comme un pistolet qui tire à blanc, mais il est tout de même irrésistible - peut-être précisément pour cette raison.

61 Si la persona de Grant associe son charme à la performance et à la mascarade, cela tient en partie à son visage apparemment sans âge, qu'il semble emprunter au portrait de Dorian Gray. Dès ses débuts aux côtés de Mae West dans les années 1930, sa beauté était la source de sa valeur en tant que star; dans l'après-guerre, il devint légendaire pour sa jeunesse éternelle - toujours bronzé, toujours séduisant, toujours le même. Pour reprendre les mots de Richard Schickel, «alors qu'il était dans sa cinquantaine, et qu'il avait toujours l'air d'avoir la quarantaine - alliant une maturité élégante et sereine à une allégresse intacte il a juste cessé de vieillir. Il a arrêté purement et simplement. C'est en tout cas l'impression qu'il donnait au public» (9). La beauté irrésistible de Grant n'était pas entamée par l'âge mais continuait à attirer le regard des femmes, dans ses films comme dans la salle, de sorte que c'est souvent elles, et non lui, qui avait 
l'initiative dans le rapport de séduction, quelle que soit la différence d'âge. Dans les deux films d'Hitchcock des années 1950 où Grant est la star principale - La Main au collet (To Catch a Thief, 1955) et La Mort aux trousses -, la blonde indifférente, sophistiquée et plus jeune que lui, finit par être assez excitée par sa beauté pour passer outre les conventions et prendre l'initiative dans le rapport de séduction. Cela illustre à quel point «Cary Grant» était synonyme de masculinité désirable et donc d'excitation sexuelle chez les femmes.

Les films que Grant tourne après-guerre montrent qu'ils ont conscience de cette dimension de sa persona en faisant allusion verbalement et visuellement à sa beauté. Lui conférant un glamour habituellement réservé aux stars féminines, ses films de la période soulignent son charme masculin dans les gros plans qui l'introduisent et donnent le même statut à son visage qu'à celui de la star féminine. Son visage bien connu fut même régulièrement l'objet de clins d'œil, comme dans la scène du train de La Mort aux trousses, lorsque Roger et Eve évoquent le fait que son visage semble familier à beaucoup de gens. L'humour repose ici sur l'interaction entre la signification de leur échange dans la diégèse et les références extradiégétiques qui parsèment le dialogue. Tout en établissant le fait qu'Eve a identifié Roger sur la photo publiée dans les journaux, et doit donc prendre en compte le danger qu'il représente en tant que meurtrier présumé, le dialogue fait allusion à l'effet rassurant et excitant du visage familier de Cary Grant dans un film romantique comme celui-ci. Quand Eve déclare que Roger peut séduire des femmes qui ne le connaissent pas, il s'agit d'une allusion à la réputation d'amant idéal dont Grant jouit à l'écran, et cette référence est ensuite approfondie lorsqu'Eve assimile son attractivité de star à sa capacité à faire vendre, comme si elle soulignait la marchandisation du célèbre visage de l'acteur. Une scène ultérieure fera encore plus explicitement allusion au fait que Grant est alors considéré comme la star masculine par excellence. Il s'agit du moment où Roger se glisse par la fenêtre d'une chambre d'hôpital occupée par une femme qui lui dit deux fois " arrêtez ", la deuxième fois après avoir mis ses lunettes pour mieux le regarder, de sorte que cette répétition de "arrêtez » signifie « restez ». Contrairement à Curtis qui doit manipuler Marilyn Monroe pour qu'elle se montre entreprenante dans Certains l'aiment chaud, Grant, lui, n'a pas besoin de duper une femme pour obtenir le même effet; dans ses films, il lui suffit de la laisser le regarder.

La Mort aux trousses met également en avant le célèbre visage de Grant à travers de nombreux gros plans, qui attirent régulièrement l'attention sur son éternelle jeunesse. La description de la performance de Grant que l'on trouve dans la critique du Hollywood Reporter, tient pour acquis le fait que Grant est là pour être regardé : «Il apparaît dans une merveilleuse série de gros plans (...), il reste fascinant sans avoir besoin de faire quelque chose (...), [dans la scène de l'attaque de l'avion] on a plus peur pour lui que pour une douzaine de Jeanne d'Arc condamnées au bûcher. Les femmes seront attirées par lui à chaque seconde, notamment dans la scène de l'hôpital où il n'est vêtu que d'une serviette de bain» (Moffitt). Outre cette mise en valeur de sa beauté par la caméra, le style de jeu extrêmement maniéré de Grant repose, dans La Mort aux trousses comme dans ses autres films, sur un ensemble d'effets dramatiques prévisibles (ce qui ne veut pas dire déplaisants ${ }^{26}$ ). En conséquence, sa diction, son sourire, ses réactions à retardement et ses gaffes - des ingrédients célèbres de son jeu qui sont mis en avant dans La Mort aux trousses, comme dans la scène à Glen Clove où Roger parvient à échapper à Vandamm - contribuent, avec sa fameuse élégance, à souligner le fait que sa 
persona masculine est plus théâtrale qu'authentique, sans pour autant diminuer son sex-appeal ou sa valeur au box-office.

Avec sa persona d'amant suave, accompli et éternellement jeune, construction artificielle et théâtrale d'une identité masculine séduisante, Cary Grant était la star idéale pour ce film dans lequel les mascarades identitaires et la performativité de genre sont des motifs centraux. La plupart des films tournés par Grant après-guerre le montrent, d'une manière ou d'une autre, adopter une sorte de déguisement qui fait plus ou moins illusion, pour une raison plus ou moins convaincante, et dans un but plus ou moins innocent. Grant se livre bien sûr à une mascarade dans certains de ses premiers films : dans L'Impossible Monsieur Bébé (Bringing Up Baby, 1938) par exemple, il doit cacher son identité à la tante de Katharine Hepburn ; et dans Pile ou Face (Mr. Lucky, 1943), son personnage commence par adopter le nom d'un ami grec décédé pour éviter la conscription, puis se livre à une mascarade supplémentaire pour escroquer une mondaine (Laraine Day). Mais les déguisements de ce type deviennent encore plus fréquents dans ses films ultérieurs. Outre le fait d'être pris pour un espion dans La Mort aux trousses, les raisons qui justifient ses mascarades sont diverses et souvent assez tirées par les cheveux, ce qui souligne le caractère réflexif de ces jeux de rôle, comme lorsqu'il se fait passer pour un adolescent dans Deux sœurs vivaient en paix (The Bachelor and the Bobby-Soxer, 1947), pour une mariée dans Allez coucher ailleurs (I Was a Male War Bride, 1949), pour un adolescent et un enfant dans Chérie, je me sens rajeunir (Monkey Business, 1952), pour un homme d'affaires de l'Oregon dans La Main au collet (To Catch a Thief, 1955), ou pour un homme marié dans Indiscret (Indiscreet, 1958), une série d'impostures culminant avec le personnage aux multiples masques qu'il incarne face à Audrey Hepburn dans Charade (1963), son dernier grand rôle romantique.

La récurrence des mascarades dans les films que Grant tourne dans les années 1950 est intimement liée à la disparité croissante entre son âge et les rôles romantiques qu'il continue de tenir. Cependant, contrairement à ce que sous-entend Certains l'aiment chaud avec sa blague sur l'impuissance, il s'agit moins par là de dissimuler une forme de défaillance masculine (la diminution des performances sexuelles chez les hommes d'âge mûr mise en évidence par Kinsey) que de renouer avec la fluidité de genre de ses comédies screwball des années 1930, comme Cette sacrée vérité (The Awful Truth, 1937) ou L'Impossible Monsieur Bébé27. À la fin des années 1950, la facilité avec laquelle la persona de Grant oscille entre les termes d'oppositions apparemment rigides - non seulement féminin/masculin mais aussi britannique/américain, distingué/ordinaire, jeune/ vieillissant - les faisait apparaître comme plus contradictoires que jamais : d'une part, à la différence de beaucoup d'autres acteurs comiques plus secondaires, il incarnait le raffinement et la distinction sans paraître efféminé ou élitiste; d'autre part, à la différence de beaucoup d'autres stars masculines étrangères, il exploitait son image d'acteur romantique au charme impérissable tout en conservant une image d'homme viril et d'Américain de classe moyenne qui le rapprochait des Wayne, Stewart, Cooper et Bogart.

Contrairement à la persona de ces autres stars masculines, celle de Grant risquait toujours de contredire l'effort par lequel l'imaginaire dominant des années 1950 opposait la virilité à l'efféminement dans le but d'authentifier la masculinité, de l'extraire du domaine de la représentation pour en faire l'origine de toutes les autres performances de genre. Cette célébration enjouée de la théâtralité aux dépens de l'authenticité, enracine sa persona séduisante de beau gosse hollywoodien ultime dans 
une performance de la masculinité comparable à celle de Roger Thornhill - ou même de George Kaplan. Ainsi, quand La Mort aux trousses met en scène Cary Grant se livrant à une mascarade, dans les scènes où Roger adopte la persona de Kaplan, il redouble la performance de la star à l'intérieur de la diégèse, et brouille ainsi la frontière qui sépare la performance du personnage et celle de l'acteur. En fait, dans la mesure où "Cary Grant » est aussi un nom donné à une persona fabriquée de toutes pièces, il y a peut-être encore plus de ressemblance entre l'accession d'Archie Leach au statut de star hollywoodienne et l'invention de George Kaplan qu'entre l'image théatrale de la star et le jeu de rôle auquel se livre Roger Thornhill ${ }^{28}$. "George Kaplan » et "Cary Grant " sont deux personae qui ont d'autant plus de pouvoir et de crédibilité qu'elles sont des inventions intégrales et couronnées de succès.

\section{Masculinité au bord du gouffre}

67 La similarité entre « Kaplan » et " Grant » permet saisir en quoi l'invention de l'espion imaginaire dans La Mort aux trousses, a la même fonction que l'invention de « Madeleine Elster » dans Sueurs froides. Pour reprendre les mots de Scottie dans la scène finale de ce film, la « Madeleine » de ses désirs se révèle être la première « copie » ou « imitation » qui met en lumière le fait que le genre se fonde sur la mascarade. Quand Sueurs froides lève le voile sur la blondeur de Madeleine pour mettre en lumière la place qu'elle occupe dans une chaîne de contrefaçons, il confirme ce que la persona de Kim Novak signifie, à savoir que la féminité est une performance que le star-système hollywoodien célèbre sous le nom de glamour féminin. Quand La Mort aux trousses laisse Roger être pris pour Kaplan, cette chaîne de contrefaçons masculines produit une révélation similaire, mais parce qu'elle concerne la masculinité, elle est beaucoup plus troublante. La masculinité de Roger Thornhill - comme celle de George Kaplan ou de Cary Grant est autant une mascarade performative que la féminité ; sa masculinité, comme la féminité d'Eve, est une persona genrée qui n'a pas d'origine au-delà de la représentation et doit donc constamment se reconstituer dans une représentation. La masculinité en crise mise en scène sur l'affiche du film - l'image de Cary Grant en train de tomber, avec le sol qui se dérobe sous ses pieds - est l'illustration parfaite de la position précaire de la «masculinité » dans cette chaîne de représentations de genre " contrefaites ». De manière assez significative, le Grant auquel l'affiche fait référence est lui-même une représentation, une copie de copie, puisqu'il s'agit du moment où Roger se prête au jeu de la performance avec le plus d'enthousiasme : la scène du faux meurtre. Comme le souligne l'inconsistance de George Kaplan dans le film, la représentation ne fournit effectivement à la masculinité qu'un fondement très précaire.

Traversé à de nombreux niveaux par les motifs de la mascarade et de la performance identitaire, La Mort aux trousses remet en question le fait que la masculinité puisse être considérée comme une identité culturelle cohérente et bien définie, pour ne pas dire authentique. C'est la raison pour laquelle la conclusion du film déploie tant d'efforts pour restaurer les idéologies de genre dominantes à cette période. Mais parce que ces efforts dévoilent le système idéologique complexe qui conduit Roger sur le chemin du mariage, de la maturité et de la masculinité adulte, la fin de La Mort aux trousses peut aussi être vue comme une performance de genre supplémentaire. Dans son célèbre plan final du train pénétrant dans le tunnel sur le chemin du retour à Grand Central Station, 
le film fait un clin d'œil encore plus osé aux nombreux impératifs idéologiques qui motivent sa résolution. En faisant cette lecture, je ne veux pas suggérer que La Mort aux trousses conteste ou remet en question le statut hégémonique de la masculinité de Roger Thornhill; ce film n'est en aucun cas utopique ou révolutionnaire dans ses intentions. Mais je soutiens qu'à travers son tableau historique subtil de l'idéologie de la performance qui est au fondement des personae de son héros, de l'ennemi fictif de ce dernier, et de la star qui l'incarne, La Mort aux trousses attire notre attention sur la mascarade qui est à la base des identités de genre dans notre culture, que ce soit la masculinité ou la féminité. Je fais de cette hypothèse le point de départ de mon étude du cinéma hollywoodien des années $1950^{29}$, de même que je tiens la relation de Roger Thornhill à George Kaplan - et à Cary Grant - comme emblématique du statut de la masculinité dans les films de cette période.

\section{BIBLIOGRAPHIE}

ALLoway Lawrence (1959), The Long Front of Culture, cité dans : Pacific Film Archive program notes, 14 Apr. 1991, North by Northwest file, Herrick.

ANON. (1960), « A Leap Year's Guide to 8 Types of Bachelors », Photoplay, March, p. 46-48, 87.

ANON. (1958), « 6 Ways to Rope in that Summer Romance », Photoplay, Sept., p. 27-30, 95-97.

ANON. (1957), « Freedom - New Style », Time, 27 Sept., p. 22-25.

ATTWOoD William (1958), « Why Does He Work So Hard?», Look, 4 March, p. 71-75.

BARTON Sabrina (1991), « 'Crisscross' : Paranoia and Projection in Strangers on a Train », Camera Obscura, 25-26, p. 74-100.

BISKIND Peter (1983), Seeing Is Believing : How Hollywood Taught Us to Stop Worrying and Love the Fifties, New York, Pantheon.

BRILL Leslie (1988), The Hitchcock Romance : Love and Irony in Hitchcock's Films, Princeton, Princeton University Press.

BRITTON Andrew (1986), « Cary Grant : Comedy and Male Desire », Cine-Action !, 7, Dec., p. 36-51.

BUTLER Judith (1993), Bodies That Matter : On the Discursive Limits of «Sex», New York, Routledge (traduction française : Judith BUTLER, Ces corps qui comptent. De la matérialité et des limites discursives $d u$ «sexe », Paris, Amsterdam, 2009).

BUTLER Judith (1991), « Imitation and Gender Insubordination », dans Diana FUSS (ed.), Inside/out : Lesbian Theories, Gay Theories, New York, Routledge, p. 13-31 (traduction française : Judith BUTLER, «Imitation et insubordination du genre », dans Judith BUTLER \& Gayle RUBIN, Marché au sexe, Paris, EPEL, 2001).

BUTLER Judith (1990), Gender Trouble : Feminism and the Subversion of Identity, New York, Routledge (traduction française : Judith BUTLER, Trouble dans le genre. Pour un féminisme de la subversion, Paris, La Découverte, 2005). 
CAVELL Stanley (1981), « North by Northwest », Critical Inquiry, 7, p. 761-776

CHAUNCEY George (1994), Gay New York: Gender, Urban Culture, and the Making of the Gay Male World 1890-1940, New York, Basic (traduction française : George CHAUNCEY, Gay New York (1890-1940), Paris, Fayard, 2003).

CLARK Clifford E. (1989), « Ranch-House Suburbia : Ideals and Realities », dans Lary MAY (ed.), Recasting America : Culture and Politics in the Age of Cold War, Chicago, University of Chicago Press, p. 172-191.

CORBER Robert J. (1993), In the Name of National Security : Hitchcock, Homophobia, and the Political Construction of Gender in Postwar America, Duhram, MC, Duke University Press.

COUGHLAN Robert (1956), « Changing Roles in Modern Marriage », Life, 24 Dec., p. 108-118.

DOANE Mary Ann (1991), Femmes Fatales : Feminism, Film Theory, Psychoanalysis, New York, Routledge.

DYER Richard (1988), « White », Screen, 29(4), 1988, p. 44-64 (traduction française : Richard DYER, «Blanc», Miranda [en ligne], n² 23, automne 2021, à paraître).

DYER Richard (1986b), Heavenly Bodies : Film Stars and Society, New York, St. Martin's, (traductions françaises partielles : Richard DYER, « Marilyn Monroe et la sexualité », dans Richard DYER, Le Starsystème hollywoodien, Paris, L'Harmattan, 2004, p. 149-197 ; Richard DYER, « Judy Garland et les hommes gays ", Genre en séries : cinéma, télévsion, médias [En ligne], 8, 2018).

DYER Richard (1986a[1979]), Stars, London, BFI (traduction française partielle : Richard DYER, Le Star-système hollywoodien, Paris, L'Harmattan, 2004).

EHRENREICH Barbara (1989), Fear of Falling: The Inner Life of the Middle Class, New York, Pantheon.

EHRENREICH Barbara (1983), The Hearts of Men : American Dreams and the Flight from Commitment, New York, Doubleday.

FLETCHER John (1988), « Versions of Masquerade », Screen, 29(3), p. 43-71

FRANK Lawrence K. (1955), « How Much Do We Know about Men?», Look, 17 May, p. 52-56.

GARBER Marjorie (1992), Vested Interests : Cross-Dressing and Cultural Anxiety, New York, Routledge.

GOFFMAN Erving (1959), The Presentation of Self in Everyday Life, New York, Anchor (traduction française : Erving GOFFMAN, La Mise en scène de la vie quotidienne, Paris, Minuit, 1973)

HIGHAM Charles \& MOSELEY Roy (1990[1989]), Cary Grant : The Lonely Heart, New York, Avon.

(traduction française : Charles HIGHAM \& Roy MOSELEY, Cary Grant, un cœur solitaire, Paris, S.

Messinger, 1989)

HOLMUND Chris (1993), « Masculinity as Multiple Masquerade : The 'Mature' Stallone and the Stallone Clone », dans Steven COHAN and Ina Rae HARK (ed.), Screening the Male : Exploring Masculinities in Hollywood Cinema, London, Routledge, p. 213-229

IRIGARAY Luce (1985), This Sex Which Is Not One, Ithaca, Cornell University Press (texte original : Luce IRIGARAY, Ce sexe qui n'en est pas un, Paris, Minuit, 1977).

KAEL Pauline (1982), 5001 Nights at the Movies : A Guide from A to Z, New York, Holt. KAPSIS Robert E. (1992), Hitchcock: The Making of a Reputation, Chicago, University of Chicago Press. KARDINER Abram (1963), « The Flight from Masculinity », in RUITENBEEK Hendrik M. (ed.), The Problem of Homosexuality in Modern Society, New York, Dutton, p. 17-39 
KLAPP Orrin E. (1962), Heroes, Villains, and Fools : The Changing American Character, Englewood Cliffs, NJ, Prentice-Hall.

LEARS Jackson (1989), « A Matter of Taste : Corporate Cultural Hegemony in a Mass-Consumption Society », dans Lary MAY (ed.), Recasting America : Culture and Politics in the Age of Cold War, Chicago, University of Chicago Press, p. 38-57.

LEFF Leonard J. (1983), « Hitchcock at Metro », Western Humanities Review, 37, p. 97-127.

LEONARD George B. Jr. (1958), « Why Is He Afraid to Be Different? », Look, 18 Feb., p. 95-102

LEHMAN Ernest (1972), North by Northwest, New York, Viking.

MAY Elaine Tyler (1988), Homeward Bound: American Families in the Cold War Era, New York, Basic.

MODLESKI Tania (1989), The Women Who Knew Too Much : Hitchcock and Feminist Theory, New York, Routledge (traduction française : Tania MODLESKI, Hitchcock et la théorie féministe. Les femmes qui en savaient trop, Paris, L'Harmattan, 2002).

MODLESKI Tania (1991), Feminism Without Women : Culture and Criticism in a « Postfeminist » Age, New York, Routledge.

MOFFITT Jack (1959), Review of North by Northwest, Hollywood Reporter, 30 June, p. 3.

MOSKIN J. Robert (1958), « Why Do Women Dominate Him ? », Look, 4 Feb., p. 77-80.

NAREMORE James (1988), Acting in the Cinema, Berkeley, University of California Press (traduction française : James NAREMORE, Acteurs : le jeu de l'acteur de cinéma, Rennes, Presses Universitaires de Rennes, 2014).

OAKLEY J. Ronald (1990), God's Country : America in the Fifties, New York, Dembner.

PACKARD Vance (1960[1959]), The Status Seekers, Harmondsworth, Pelican (traduction française : Vance PACKARD, Les Obsédés du standing, Paris, Calmann-Lévy, 1962).

PERCHUK Andrew, and Helaine POSNER (ed.) (1995), The Masculine Masquerade : Masculinity and Representation, Cambridge, MIT Press.

POWE (1959), Review of North by Northwest, Daily Variety, 30 June, p. 3.

RIVIERE Joan (1929), « Womanliness as a Masquerade », in BURGIN Victor, DONALD James, and KAPLAN Cora (ed.), Formations of Fantasy, London, Methuen, 1986, p. 35-44 (traduction française : Joan RIVIERE, « Féminité mascarade », dans Marie-Christine Hamon (ed.), Féminité mascarade : études psychanalytiques, Paris, Seuil, 1994).

ROGIN Michael Paul (1987), Ronald Reagan, the Movie and Other Episodes in Political Demonology, Berkeley, University of California Press (traduction française partielle : Michael RoGIN, Les Démons de l'Amérique : essais d'histoire politique des États-Unis, Paris, Seuil, 1998).

ROTHмAN William (1988), The «I I of the Camera : Essays in Film Criticism, History, and Aesthetics, New York, Cambridge University Press.

RUITENBEEK Hendrik M. (1963), « Men Alone : The Male Homosexual and the Disintegrated Family ", dans Hendrik M. Ruitenbeek (ed.), The Problem of Homosexuality in Modern Society, New York, Dutton, p. 80-93.

SCHICKEL Richard (1983), Cary Grant : A Celebration, Boston, Little.

SPIGEL Lynn (1992), Make Room for TV : Television and the Family Ideal in Postwar America, Chicago, University of Chicago Press. 
SРото Donald (1993[1983]), The Dark Side of Genius : The Life of Alfred Hitchcock, New York, Ballantine (traduction française : Donald SРото, La face cachée d'un génie : La vraie vie d'Alfred Hitchcock, Paris, Albin Michel, 1989).

TRUFFAUT François (1984), Hitchcock, New York, Simon (texte original : François TRUFFAUT, Hitchcock/Truffaut, Paris, Gallimard, 1993[1966]).

WILson George M. (1986), Narration in Light: Studies in Cinematic Point of View, Baltimore, Johns

Hopkins University Press.

wood Robin (1989), Hitchcock's Films Revisited, New York, Columbia University Press.

\section{NOTES}

1. Il est intéressant de noter que cette scène ne fut pas un tournant pour le film uniquement pour cette raison, puisque "l'idée de Eve tirant sur Thornhill avec des balles à blanc " semble avoir joué un rôle de catalyseur pour Ernest Lehman, en relançant son processus d'écriture à la fin de l'année 1957, alors qu'un blocage l'empêchait d'achever le dernier quart du scénario (Leff 105).

2. Le terme latin «persona » désignait le masque de l'acteur. Dans cet article, l'auteur utilise le terme anglais de "persona " pour désigner à la fois l'image que les gens en général projettent en public (comme c'est le cas dans cette phrase), et l'image médiatique des stars, qui se nourrit entre autres des rôles incarnés à l'écran, des photographies promotionnelles ou des discours tenus sur la star dans la presse (cf. notamment la partie «Performer les genres », qui fait le lien entre ces deux sens). Afin de conserver la multiplicité de ces connotations, et dans la mesure où l'usage de ce terme est aujourd'hui répandu dans le champ de l'étude des stars (star studies) en contexte francophone, je conserve ce mot dans la traduction française (NdT).

3. Tania Modleski suggère que, dans Sueurs froides comme dans La Mort aux trousses, la «crainte des hauteurs » est associée à la "féminité » - et plus précisément à la féminité définie, par rapport à la masculinité, comme un manque symbolique (Modleski 1989, 90).

4. Wood a reproduit son livre de 1965 à l'intérieur de Hitchcock's Film Revisited (p. 55-236). Pour son analyse de La Mort aux trousses comme récit d'accès à la maturité, voir p. 131-141.

5. Terme désignant la personne (ici, l'homme) qui gagne l'argent permettant de nourrir la famille $(\mathrm{NdT})$.

6. Dans l'ordre de publication, les trois articles étaient écrits par Moskin, Leonard et Attwood. Ils ont été ensuite republiés la même année sous la forme d'un livre intitulé The Decline of the American Male, by the editors of Look (New York, Random House, 1958).

7. Edward Gibbon est un historien anglais auteur de The History of the Decline and Fall of the Roman Empire (Histoire de la décadence et de la chute de l'Empire romain), ouvrage en plusieurs volumes originellement publiés entre 1776 et $1789(\mathrm{NdT})$.

8. L'expression utilisée dans le texte original (" picked up») signifie à la fois "prendre » (quelqu'un pour l'emmener ailleurs) et « draguer».

9. L'expression « domestic security » joue ici sur le double sens de «domestic » qui renvoie à la fois à la sphère domestique (le foyer) et, en politique, aux affaires intérieures (par opposition aux affaires étrangères) $(\mathrm{NdT})$.

10. Geoffrey M. Shurlock, Letters to Joseph Vogel, 2 Oct. 1958 and 16 Oct. 1958 (North by Northwest PCA file).

11. «Tall, dark and handsome » est une façon commune de désigner des hommes séduisants (NdT).

12. Le blockbusting est une pratique de promoteurs et d'agent immobiliers visant à racheter la maison de propriétaires blancs à bas prix en agitant le spectre de l'installation de minorités raciales dans leur quartier $(\mathrm{NdT})$. 
13. La «fonction spéculaire» en question désigne le fait d'être un miroir des fantasmes masculins, et de renvoyer ainsi aux hommes l'image de leur propre désir. Cf. un peu plus haut, le passage où l'auteur, se référant aux analyses de Sabrina Barton, explique que l'image fétichisée de la blonde "offre au public une projection du désir masculin (a spectularization of male desire)» $(\mathrm{NdT})$.

14. George M. Wilson lit également le film comme une défense de l'illusionnisme, mais au lieu d'historiciser ce thème, comme je le fais, il l'interprète comme un exemple de métacinéma hitchcockien : une «apologie ironique du type d'art illusionniste - plus précisément, de cinéma illusionniste - dont les films d'Hitchcock sont un exemple paradigmatique » (Wilson 64). Mais ce film est autant un scénario d'Ernest Lehman qu'un classique d'Alfred Hitchcock. En plaçant sciemment Roger dans le milieu des médias, La Mort aux trousses s'apparente fortement au précédent film de Lehman, Le Grand Chantage (Sweet Smell of Success, 1957), un film noir dépeignant la vie nocturne de chroniqueurs et d'agents de publicité. On peut ainsi raisonnablement penser que l'apport de Lehman à ce scénario préoccupé par les questions d'illusion et de performance a sans doute contribué à donner à La Mort aux trousses cette teneur historique sur laquelle j'insiste.

15. 20th Century-Fox Press Release, Oct. 1957, Grant file, Herrick. Ce communiqué de presse faisait partie de la campagne promotionnelle du film Elle et lui (An Affair to Remember, 1957), dans lequel la star portait «ses propres vêtements", mais cette description de son sens de la mode peut aussi bien s'appliquer à La Mort aux trousses. C'était d'ailleurs un texte standard puisqu'il apparaît également dans un communiqué de presse de 1952.

16. Naremore commente la manière dont la couleur grise des vêtements de Roger a retenu l'attention de nombreux fans du film, bien que son analyse porte avant tout sur la manière dont le costume renforce la dimension corporelle du jeu d'acteur de Grant (Naremore : 214-216).

17. Dans son ouvrage The Lonely Crowd (publié aux États-Unis en 1950 et traduit en français sous le titre La foule solitaire en 1964), David Riesman cherche à déterminer le type de caractère social correspondant à l'entrée de la société états-unienne dans une phase de prospérité (l'«affluent society»). Il qualifie ce modèle de comportement d'«extro-déterminé (other-directed)» afin d'insister sur le rôle essentiel joué par les pairs dans la détermination de l'attitude de l'individu (leur approbation étant le seul critère de réussite), et le distingue de deux autres modèles correspondant à des stades antérieurs de la société : l'individu déterminé par la tradition (tradition-directed) et l'individu « intro-déterminé » (inner-directed) (NdT).

18. L'expression « the organization man » est utilisée par William $H$. Whyte dans son livre du même nom (publié en 1956 aux États-Unis) pour décrire ce qu'il identifie comme la prééminence nouvelle (notamment au sein des entreprises privées) d'une "éthique sociale " qui aurait supplanté selon lui l'«éthique protestante» traditionnelle, et pour laquelle le groupe, l'organisation, l'entreprise, primerait sur l'individu (NdT).

19. Sur ce point, voir également Butler 1991.

20. Lorsqu'il dit que le genre est une représentation «culturellement perçue comme mimétique ", l'auteur fait allusion à la perception du genre comme dérivé d'un fondement naturel (le sexe), ou, en d'autres termes, à « la croyance en une relation mimétique du genre au sexe par laquelle le genre reflèterait le sexe ou serait limité par lui d'une autre manière » (Judith Butler, Gender Trouble, New York, Routledge, 2007, p. 9) (NdT).

21. Les articles majeurs qui appliquent la théorie de Riviere au cinéma, auxquels je fais allusions ici, sont «Film and the Masquerade : Theorizing the Female Spectator» (1982) et « Masquerade Reconsidered : Further Thoughts on the Female Spectators " (1988-89) de Mary Ann Doane, tous deux republiés dans Femmes Fatales (p. 17-43) ; et Fletcher. Voir également Irigaray, qui décrit la mascarade féminine comme un effacement «imposé aux femmes par les systèmes masculins de représentation» (84), et la voit également comme une forme d'imitation, un «jeu avec la mimésis » (76). 
22. Voir la critique d'Holmlund sur les limites de la mascarade en tant que conceptualisation de la sexualité masculine (216-219). Pour une vue d'ensemble multidisciplinaire des travaux qui vont au-delà de la théorie de Riviere pour conceptualiser la masculinité comme une mascarade, voir l'introduction de Harry Brod dans Perchuk and Posner (13-19). L'ensemble des chapitres du volume, comprenant une ancienne version de ce chapitre, illustrent d'ailleurs le rôle de plus en plus central que joue la mascarade en tant que cadre d'analyse de la masculinité dans la critique culturelle et les représentations visuelles.

23. Voir également Butler, 1990 : 136-137 ; Butler, 1991 : 24 ; Butler, 1993 : 1-16. Modleski propose de distinguer " les concepts de "performatif" et de mascarade ", en définissant la seconde comme une compensation de l'usurpation féminine d'un pouvoir masculin (conformément à la théorie de Riviere), laquelle se désexualiserait ainsi en quelque sorte, là où le premier ne contiendrait «pas un tel reniement» (Modleski, 1991: 54), mais je pense qu'il est préférable de ne pas faire une distinction aussi nette entre "performance» et "mascarade» afin de reconnaître leur imbrication : l'effet de la performativité est la mascarade, et la mascarade se constitue à travers la performance, ce qui en fait autant une action qu'un objet (ou une persona).

24. "Ils sont commodes en voyage parce qu'ils sèchent vite", aurait expliqué Grant au journaliste Joe Hyams environ cinq mois avant la sortie de La Mort aux trousses (Higham et Moseley : 280).

25. Les explications répandues de la crise de la masculinité de la période, comme les articles cités plus haut sur le déclin de l'homme américain, insistaient sur la nécessité de prendre en compte le problème de l'impuissance pour comprendre les pressions sociales et genrés subies par les hommes. J'étudie en détails l'influence des rapports Kinsey sur les représentations de la masculinité dans le chapitre suivant ("The "Paradox" of Hegemonic Masculinity »).

26. Pour une analyse plus détaillée de la technique qui sous-tend le jeu d'acteur apparemment naturel de Grant dans La Mort aux trousses, voir Naremore : 213-235.

27. Dans ma référence aux comédies screwball, je reprends à mon compte l'analyse d'Andrew Britton sur la «féminité masculine » de Grant (50). Ces films, explique Britton, « utilisent Grant pour représenter un type de masculinité qui est valorisée et présentée comme séduisante en tant qu'elle partage des caractéristiques genrées avec les femmes » (43).

28. Naremore remarque également que Kaplan et Roger sont tous les deux semblables à la star Cary Grant en tant qu'identités fabriquées (221-222).

29. L'auteur fait ici allusion à son livre Masked Men: Masculinity and the Movies in the Fifties, dont ce texte sur La Mort aux trousses constitue le chapitre inaugural (NdT).

\section{AUTEURS}

\section{STEVEN COHAN}

Steven Cohan is Professor Emeritus. He taught courses and supervised graduate research in film studies, popular culture, gender and sexualities, and cultural studies. His books include Telling Stories : A Theoretical Analysis of Narrative (1988, co-authored with Linda M. Shires), Screening the Male (1993, co-edited with Ina Rae Hark), The Road Movie Book (1997, co-edited with Ina Rae Hark), Masked Men : Masculinity and the Movies in the Fifties(1997), Hollywood Musicals, The Film Reader (2001), Incongruous Entertainment: Camp, Cultural Value, and the MGM Musical (2005), CSI : Crime Scene Investigation (2008) The Sound of Musicals (2010), Hollywood by Hollywood (2018), and Routledge Film 
Guidebooks : Hollywood Musicals (2019). His essays have appeared in Camera Obscura,Screen, and Cinema Journal as well as many anthologies. Since retiring he has written essays on Danny Kaye's queer persona, The Boys in the Band, Billy Wilder's apartment plots, Marilyn Monroe biopics, the cold war cycle of musicals set in Paris, Bob Hope's comedian musicals, Judy Garland as a cult star, Esther Williams's Latin lovers, Queer Hollywood Musicals of the 1940s, the 1937 A Star Is Born, and Fosse/Verdon. 